\title{
A New Ru-Catalyzed Cascade Reaction Forming Polycyclic Cyclohexadienes from 1,6-Diynes and Alkenes
}

\author{
Jesús A. Varela, Silvia G. Rubín, Carlos González-Rodríguez, Luis Castedo, Carlos Saá* \\ Departamento de Química Orgánica y Unidad Asociada al CSIC, Facultad de Química, Universidad de \\ Santiago de Compostela, 15782 Santiago de Compostela, Spain
}

\section{Supporting Information}

Index

$\begin{array}{ll}\text { I. General } & \text { S2 }\end{array}$

II. Preparation of starting materials and catalysts $\quad$ S2

III. General procedure for the Ru-catalyzed addition of alkenes 2 to 1,6-diynes 1 followed by $6 e^{-} \pi$ electrocyclization

$I V$. Isolation of hexatrienes 3 and ${ }^{1} H$-NMR experiments for the thermal $6 e^{-} \pi$ electrocyclization of 3 to cyclohexadienes 4.

$\begin{array}{ll}\text { V. Preliminary DFT calculations } & \text { S7 }\end{array}$

$\begin{array}{ll}\text { VI. Spectral data } & \text { S10 }\end{array}$

$\begin{array}{ll}\text { VII. References } & \text { S16 }\end{array}$

$\begin{array}{ll}\text { VIII. Spectra } & \text { S17 }\end{array}$ 
Dry DMF (Fluka) was used as received. All other solvents and reagents were used as received, unless otherwise noted. All reactions were carried out under an atmosphere of argon in flame-dried glassware with magnetic stirring, unless otherwise indicated.

\section{Preparation of starting materials and catalysts}

2,2-Diprop-2-ynyl malonic acid dimethyl ester (1a) was prepared following published procedures. ${ }^{1}$ Catalyst $\left[\mathrm{Cp} * \mathrm{Ru}\left(\mathrm{CH}_{3} \mathrm{CN}\right)_{3}\right] \mathrm{PF}_{6}$ was also prepared following published procedures. ${ }^{2,3}$

III. General procedure for the Ru-catalyzed addition of alkenes 2 to 1,6-diynes 1 followed by $6 e^{-} \pi$ electrocyclization.

\section{Conditions A:}

Trimethyl 6,7-dihydro-1H-indene-2,2,5(3H)-tricarboxylate (4a). To a stirred solution of $\left[\mathrm{Cp}^{*} \mathrm{Ru}\left(\mathrm{CH}_{3} \mathrm{CN}\right)_{3}\right] \mathrm{PF}_{6}(25 \mathrm{mg}, 10 \% \mathrm{~mol})$ and $\mathrm{Et}_{4} \mathrm{NCl}(8 \mathrm{mg}, 10 \% \mathrm{~mol})$ in DMF $(1.5 \mathrm{~mL})$ was added the alkene $2 \mathrm{a}(129 \mathrm{mg}, 0.14 \mathrm{~mL}, 1.5 \mathrm{mmol}, 3 \mathrm{eq}$ ) at room temperature. After stirring for 10 minutes, the diyne 1a (104 mg, $0.5 \mathrm{mmol}, 1$ eq.) in DMF $(2 \mathrm{~mL})$ was added to the solution over 4 hours via syringe pump. During the slow addition of the diyne 1a to the reaction flask the solution was heated at $80^{\circ} \mathrm{C}$. Stirring was continued for 1 hour after finishing the slow addition, and the reaction was quenched with saturated aqueous solution of $\mathrm{NH}_{4} \mathrm{Cl}(10 \mathrm{~mL})$ and extracted with ethyl ether $(3 \times 10$ $\mathrm{mL}$ ). The organic layers were collected, dried over anhydrous $\mathrm{Na}_{2} \mathrm{SO}_{4}$ and evaporated under vacuum. Column chromatography of the residue on silica gel using ethyl acetate/hexane (4:6) as eluents afforded 1,3-cyclohexadiene 4a in $62 \%$ yield.

\section{Conditions B:}

Dimethyl 6-(ethoxymethyl)-4,5-dihydro-1H-indene-2,2(3H)-dicarboxylate $(4 \mathrm{f})$. In a $10 \mathrm{~mL}$ roundbottomed flask was prepared, under argon, a solution of $\left[\mathrm{Cp}^{*} \mathrm{Ru}\left(\mathrm{CH}_{3} \mathrm{CN}\right)_{3}\right] \mathrm{PF}_{6}(25 \mathrm{mg}, 10 \% \mathrm{~mol})$ and $\mathrm{Et}_{4} \mathrm{NCl}(8 \mathrm{mg}, 10 \% \mathrm{~mol})$ in DMF $(1.5 \mathrm{~mL})$. After stirring for 10 minutes at room temperature the alkene $2 \mathrm{f}(430 \mathrm{mg}, 0.56 \mathrm{~mL}, 5 \mathrm{mmol}, 10 \mathrm{eq}$.) was added. After stirring for another 10 minutes, the diyne 1 a (104 mg, $0.5 \mathrm{mmol}, 1$ eq.) was added and the resulting solution was heated at $80^{\circ} \mathrm{C}$ during 2 hours. The reaction was quenched with saturated aqueous solution of $\mathrm{NH}_{4} \mathrm{Cl}(10 \mathrm{~mL})$ and extracted with ethyl ether $(3 \times 10 \mathrm{~mL})$. The organic layers were collected, dried over anhydrous $\mathrm{Na}_{2} \mathrm{SO}_{4}$ and evaporated under vacuum. Column chromatography of the residue on silica gel using ethyl acetate/hexane (2:8) as eluents afforded 1,3-cyclohexadiene $\mathbf{4 f}$ in $90 \%$ yield.

$I V$. Isolation of hexatrienes 3 and ${ }^{1} H-N M R$ experiments for the thermal $6 e^{-} \pi$ electrocyclization of 3 to cyclohexadienes 4

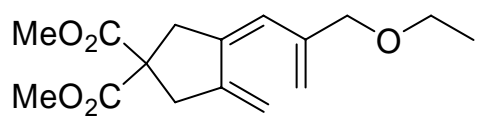

$3 \mathbf{f}$

(Z)-Diethyl 3-(2-(ethoxymethyl)allylidene)-4-methylenecyclopentane-1,1-dicarboxylate (3f): In a $10 \mathrm{~mL}$ round-bottomed flask was prepared, under argon, a solution of $\left[\mathrm{Cp} * \mathrm{Ru}\left(\mathrm{CH}_{3} \mathrm{CN}\right)_{3}\right] \mathrm{PF}_{6}(25 \mathrm{mg}$, $10 \% \mathrm{~mol})$ and $\mathrm{Et}_{4} \mathrm{NCl}(8 \mathrm{mg}, 10 \% \mathrm{~mol})$ in $\mathrm{DMF}(1 \mathrm{~mL})$. After stirring for 10 minutes at room temperature the alkene $2 \mathrm{f}(430 \mathrm{mg}, 0.56 \mathrm{~mL}, 5 \mathrm{mmol}, 10 \mathrm{eq}$.) was added to the solution, and after 
another 10 minutes, the diyne 1a (104 mg, $0.5 \mathrm{mmol}, 1$ eq.) was also added. After stirring the reaction mixture for $1.5 \mathrm{~h}$ at room temperature, the crude solution was charged directly to a column chromatography on silica gel and eluted using ethyl ether/hexane (7:3). After evaporating the eluent under vacuum in a rotavapor keeping the bath at room temperature, the hexatriene $3 \mathbf{f}$ was isolated in a $66 \%$ yield as a colorless oil.

${ }^{1} \mathrm{H}-\mathrm{NMR}\left(300 \mathrm{MHz}, \mathrm{CDCl}_{3}\right), \delta(\mathrm{ppm}): 5.90(\mathrm{~s}, 1 \mathrm{H}), 5.68(\mathrm{~s}, 1 \mathrm{H}), 5.27(\mathrm{~s}, 1 \mathrm{H}), 5.19(\mathrm{~s}, 1 \mathrm{H}), 5.05(\mathrm{~s}$, $1 \mathrm{H}), 3.96(\mathrm{~s}, 2 \mathrm{H}), 3.70(\mathrm{~s}, 6 \mathrm{H}), 3.46(\mathrm{q}, J=7.0 \mathrm{~Hz}, 2 \mathrm{H}), 3.03(\mathrm{~s}, 2 \mathrm{H}), 3.00(\mathrm{~s}, 2 \mathrm{H}), 1.18$ (t, J= 7.0 Hz, $3 \mathrm{H}) .{ }^{13} \mathrm{C}-\mathrm{NMR}$, DEPT $\left(75 \mathrm{MHz}, \mathrm{CDCl}_{3}\right), \delta(\mathrm{ppm}): 171.5(2 \times \mathrm{CO}), 142.6(\mathrm{C}), 142.3(\mathrm{C}), 137.2(\mathrm{C})$, $123.4(\mathrm{CH}), 114.6\left(\mathrm{CH}_{2}\right), 111.3\left(\mathrm{CH}_{2}\right), 71.9\left(\mathrm{CH}_{2}\right), 65.6\left(\mathrm{CH}_{2}\right), 57.0(\mathrm{C}), 52.7\left(2 \times \mathrm{CH}_{3}\right), 42.9\left(\mathrm{CH}_{2}\right)$, $42.8\left(\mathrm{CH}_{2}\right), 15.1\left(\mathrm{CH}_{3}\right)$.

${ }^{1} \mathrm{H}$-NMR experiments for the thermal $6 \mathrm{e}^{-} \boldsymbol{\pi}$ electrocyclization of $3 \mathrm{f}$ to cyclohexadiene $4 \mathrm{f}$ : A solution of hexatriene $3 \mathrm{f}$ in $\mathrm{CDCl}_{3}$ was placed in an NMR tube and heated to $50^{\circ} \mathrm{C}$. ${ }^{1} \mathrm{H}$-NMR were taken every 5 minutes observing the conversion of $\mathbf{3 f}$ into $\mathbf{4 f}$. The conversion was almost quantitative after 1 hour at $50^{\circ} \mathrm{C}$. Representatives $1 \mathrm{H}-\mathrm{NMR}$ data of the reaction are shown below. 


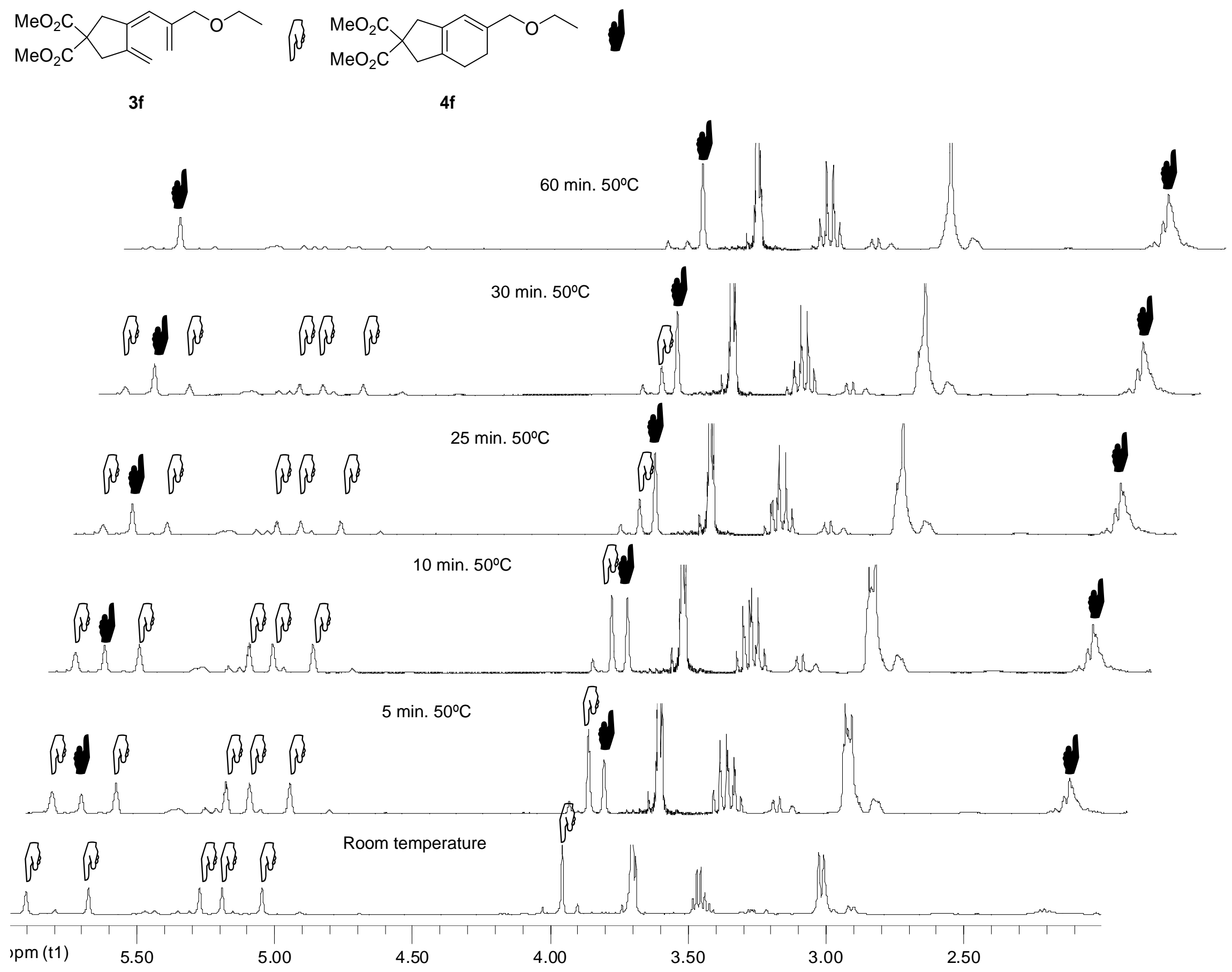


(3Z)-dimethyl<smiles>C=C1CC(C(C)=O)(C(C)=O)C/C1=C/C(=O)OC(C)=O</smiles>

3-((Z)-2,3-di(methoxycarbonyl)allylidene)-4-methylenecyclopentane-1,1-

dicarboxylate (3e): In a $10 \mathrm{~mL}$ round-bottomed flask was prepared, under argon, a solution of $\left[\mathrm{Cp} * \mathrm{Ru}\left(\mathrm{CH}_{3} \mathrm{CN}\right)_{3}\right] \mathrm{PF}_{6}(25 \mathrm{mg}, 10 \% \mathrm{~mol})$ and $\mathrm{Et}_{4} \mathrm{NCl}(8 \mathrm{mg}, 10 \% \mathrm{~mol})$ in DMF $(1 \mathrm{~mL})$. After stirring for 10 minutes at room temperature the alkene $2 \mathbf{e}(750 \mathrm{mg}, 0.65 \mathrm{~mL}, 5 \mathrm{mmol}, 10 \mathrm{eq}$.) was added to the solution, and after another 10 minutes, the diyne 1a (104 mg, $0.5 \mathrm{mmol}, 1$ eq.) was also added. After stirring the reaction mixture for $1.5 \mathrm{~h}$ at room temperature, the crude solution was charged directly to a column chromatography on silica gel and eluted using ethyl ether/hexane (6:4). After evaporating the eluent under vacuum in a rotavapor keeping the bath at room temperature, the hexatriene 3e was isolateded in a $47 \%$ yield as a pale yellow oil.

${ }^{1} \mathrm{H}-\mathrm{NMR}\left(300 \mathrm{MHz}, \mathrm{CDCl}_{3}\right), \delta(\mathrm{ppm}): 6.68(\mathrm{q}, J=2.1 \mathrm{~Hz}, 1 \mathrm{H}), 6.47(\mathrm{~d}, J=2.1 \mathrm{~Hz}, 1 \mathrm{H}), 5.12(\mathrm{t}, J=2.1$ $\mathrm{Hz}, 1 \mathrm{H}), 4.97(\mathrm{t}, J=2.1 \mathrm{~Hz}, 1 \mathrm{H}), 3.75(\mathrm{~s}, 3 \mathrm{H}), 3.74(\mathrm{~s}, 6 \mathrm{H}), 3.68(\mathrm{~s}, 3 \mathrm{H}), 3.20(\mathrm{~d}, J=2.1 \mathrm{~Hz}, 2 \mathrm{H}), 3.02$ $(\mathrm{t}, J=2.1 \mathrm{~Hz}, 2 \mathrm{H}) .{ }^{13} \mathrm{C}-\mathrm{NMR}$, DEPT $\left(75 \mathrm{MHz}, \mathrm{CDCl}_{3}\right), \delta(\mathrm{ppm}): 171.3$ (2xCO), $170.0(\mathrm{CO}), 165.7$ (CO), $144.4(\mathrm{C}), 144.3(\mathrm{C}), 143.9(\mathrm{C}), 125.5(\mathrm{CH}), 117.5(\mathrm{CH}), 113.4\left(\mathrm{CH}_{2}\right), 57.2(\mathrm{C}), 52.9\left(2 \mathrm{xCH}_{3}\right)$, $52.6\left(\mathrm{CH}_{3}\right), 51.8\left(\mathrm{CH}_{3}\right), 41.8\left(\mathrm{CH}_{2}\right), 41.4\left(\mathrm{CH}_{2}\right)$.

${ }^{1} \mathrm{H}$-NMR experiments for the thermal $6 \mathrm{e}^{-} \pi$ electrocyclization of $3 \mathrm{e}$ to cyclohexadiene $4 \mathrm{e}$ : A solution of hexatriene $3 \mathbf{e}$ in $\mathrm{CDCl}_{3}$ was placed in an NMR tube and heated to $50^{\circ} \mathrm{C} .{ }^{1} \mathrm{H}-\mathrm{NMR}$ were taken every 5 minutes observing the conversion of $\mathbf{3 e}$ into $4 \mathbf{e}$. The conversion was almost quantitative after 35 min at $50^{\circ} \mathrm{C}$. Representatives $1 \mathrm{H}-\mathrm{NMR}$ data of the reaction are shown below. 


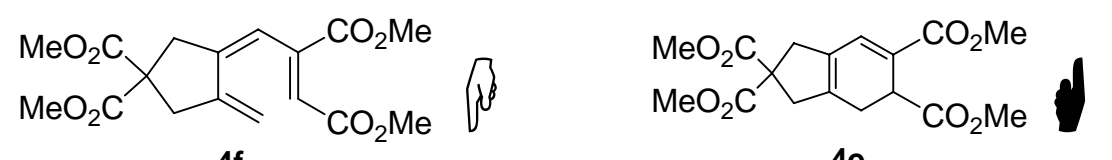

$35 \min .50^{\circ} \mathrm{C}$

$25 \min 50^{\circ} \mathrm{C}$

$\alpha \beta$

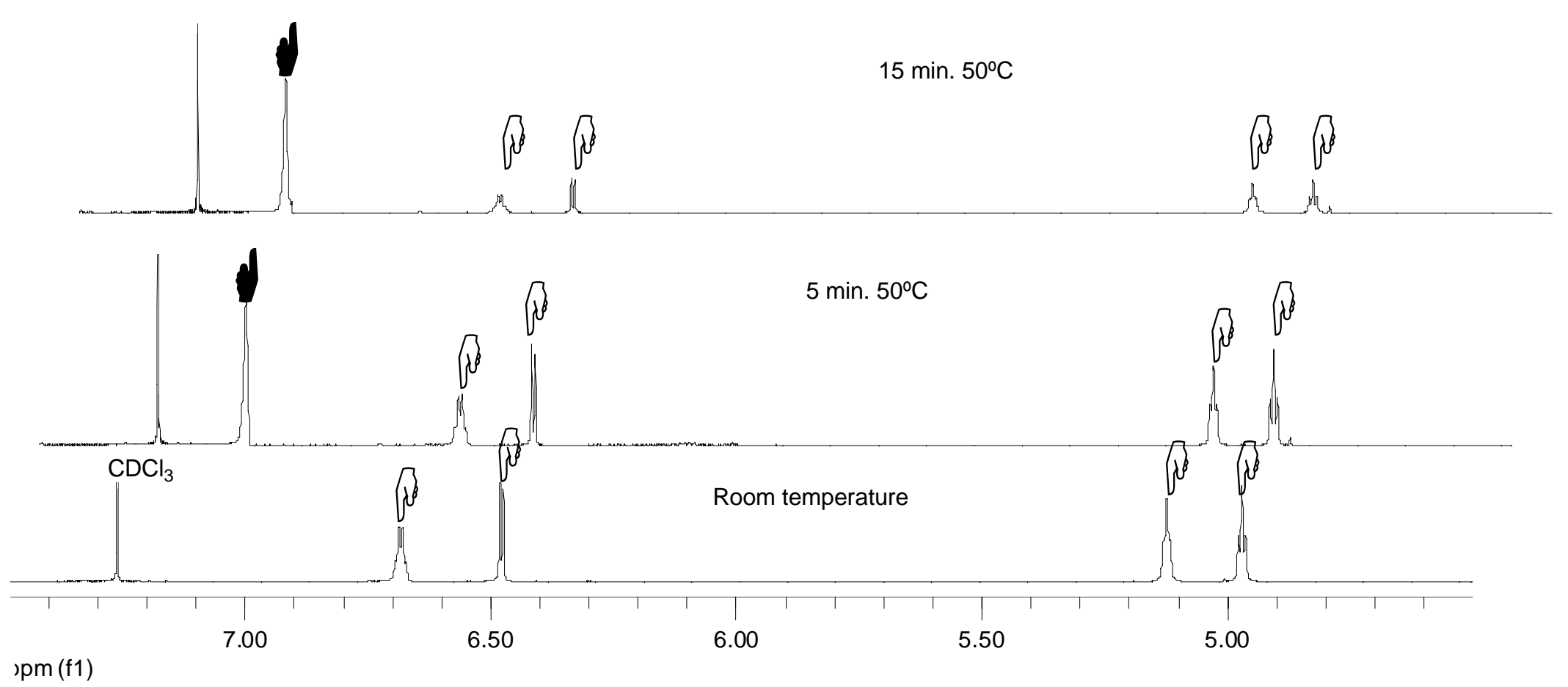




\section{Preliminary DFT calculations}

Calculations were performed with the GAUSSIAN 03 package. ${ }^{4}$ The geometries of all complexes here reported were optimized using density functional theory (DFT) at the generalized gradient approximation using the B3LYP hybrid functional. ${ }^{5}$ At these preliminary studies we have used the standard LANL2DZ basis set. ${ }^{6}$ Harmonic frequencies were calculated at B3LYP level to characterize the stationary points. Intrinsic reaction coordinate calculations (IRC) showed that the calculated TSs connect the reagents and the products.

To determinate the reaction profile for intermediate derivatives of type III, we performed density functional calculations (DFT) on ruthenacycloheptadienes III' with $R_{1}=M e, R_{2}=H$ and without the five-membered cycle (Scheme 1).

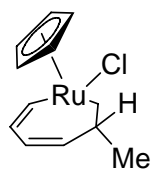

III'

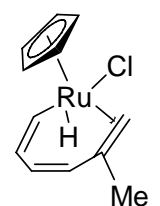

IV

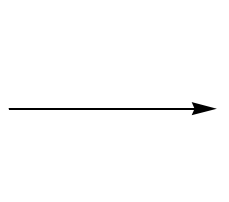

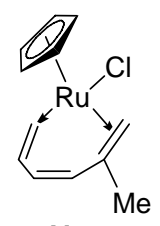

V

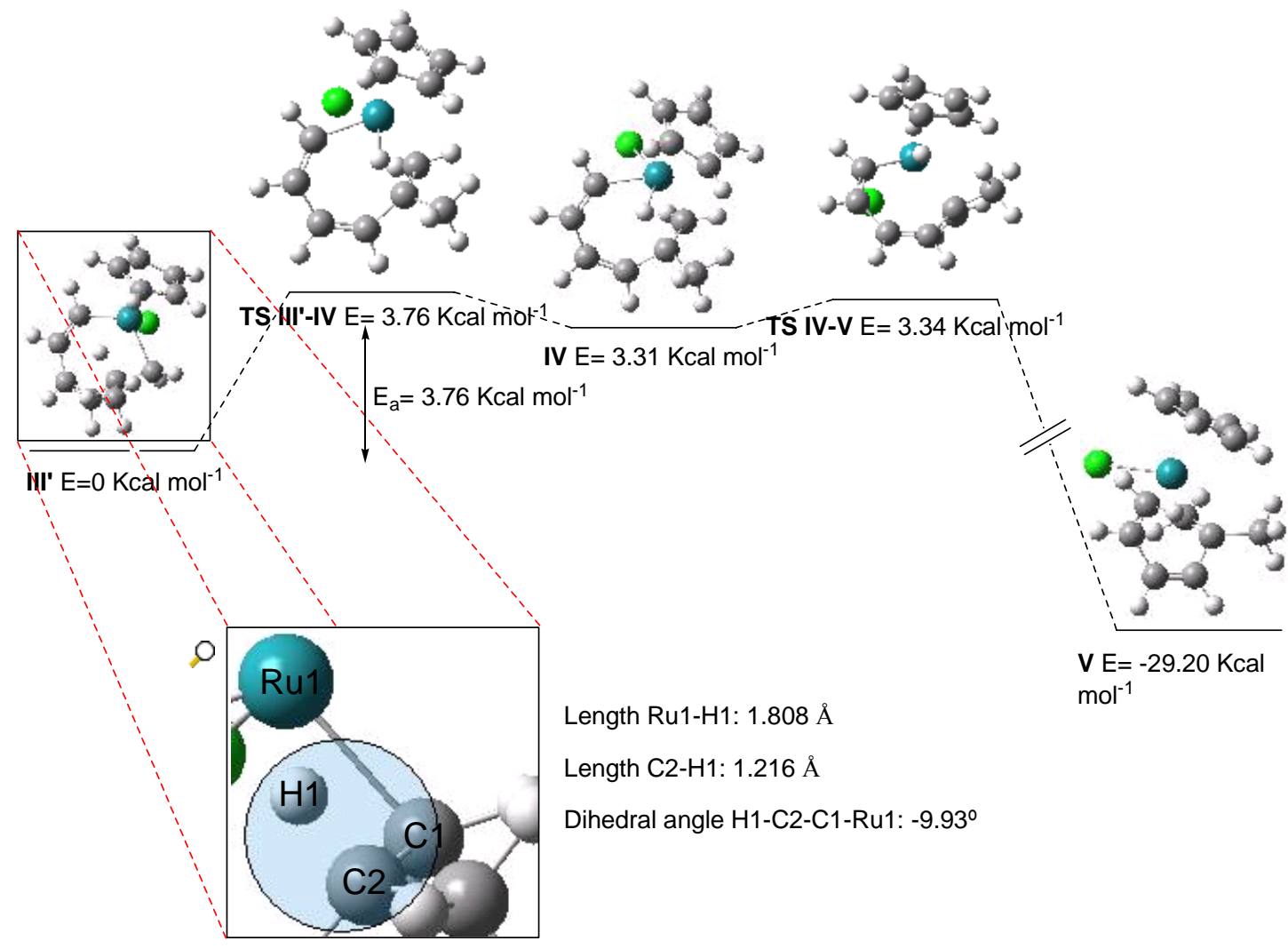

Scheme 1

According to these calculations, ruthenacycle III' presents and agostic interaction between $\mathrm{H} 1$ and Ru1 as shown by the bond length between $\mathrm{H} 1$ and $\mathrm{C} 2$, and the dihedral angle $\mathrm{H} 1-\mathrm{C} 2-\mathrm{C} 1-\mathrm{Ru} 1$ is $-9.93^{\circ}$. Calculations performed on III' led to a $\beta$-hydride elimination transition state (TSIII'-IV) of H1 which connects intermediates III'-IV and lies $3.76 \mathrm{Kcal} \mathrm{mol}^{-1}$ above III'. The following reductive elimination on intermediate IV led to the experimentally observed hexatriene of type $\mathbf{V}$ coordinated to the ruthenium and $29.20 \mathrm{Kcal} \mathrm{mol}^{-1}$ more stable than III' (Scheme 1). 
Nevertheless, calculations performed on the ruthenacycle III’, conformer of III' but $2.98 \mathrm{Kcal} \mathrm{mol}^{-1}$ less stable, led via the reductive elimination transition state TSIII'-VI, which lies $6.61 \mathrm{Kcal} \mathrm{mol}^{-1}$ above III’', to the cyclohexadienes of type VI being $40.84 \mathrm{Kcal} \mathrm{mol}^{-1}$ more stable (Scheme 2).

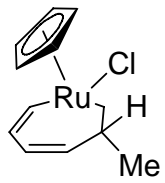

III"
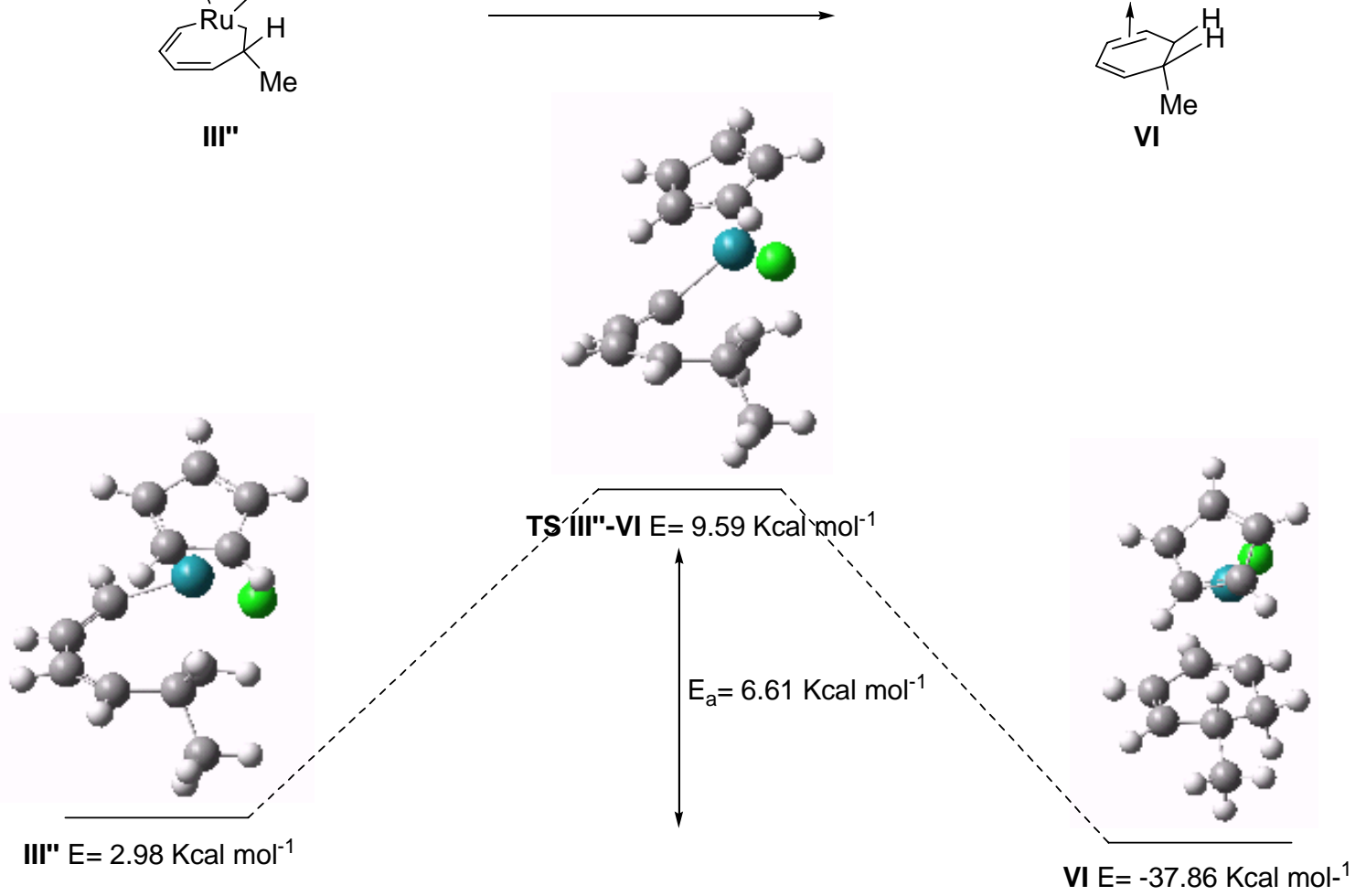

Scheme 2

All attempts to find the transition state between VII (Figure 1) and III' failed, being only able to find the transition state that led to the conformer III'. The attempts to connect directly III' with the cyclohexadiene VI via a transition state were unsuccessful too (reductive elimination process). Thus, according to these preliminary results, it seems that the first conformer formed is III', from which two possible pathways are envisioned: Pathway A, depicted in scheme 1 , affording open hexatrienes $(\beta-$ hydride elimination followed by reductive elimination, experimentally observed) and pathway B, that connects intermediate III' with III' followed by the reductive elimination shown in Scheme 2. According to the results shown in Schemes 1 and 2, pathway A seems be more favorable (conformer III' being $2.98 \mathrm{Kcal} \mathrm{mol}^{-1}$ more stable than III' and transition state for $\beta$-hydride elimination being $2.85 \mathrm{Kcal} \mathrm{mol}^{-1}$ more stable than reductive elimination).

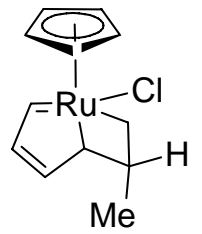

VII

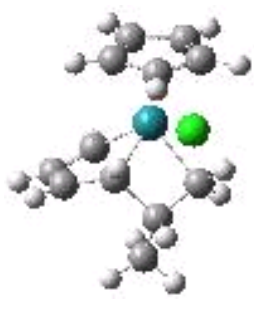

Figure 1 
When DFT calculations were performed on the intermediates from the reactions with cyclopentene 2p, a bicycloruthenacycle III'” was now available from VII' (Scheme 3). Conformer III'” has no agostic interaction between $\mathrm{H} 1$ and Ru1, being the dihedral angle H1-C2-C1-Ru1 of $-176.35^{\circ}$. Therefore, it seems that the conformation of III'", due to steric restrictions caused by the annelated five-membered ring, precludes the $\beta$-hydride elimination making more favorable the reductive elimination to the $\mathrm{Ru}$ coordinated cyclohexadiene VIII (Scheme 3). Further calculations are currently being carried in order to complete all the catalytic cycle.
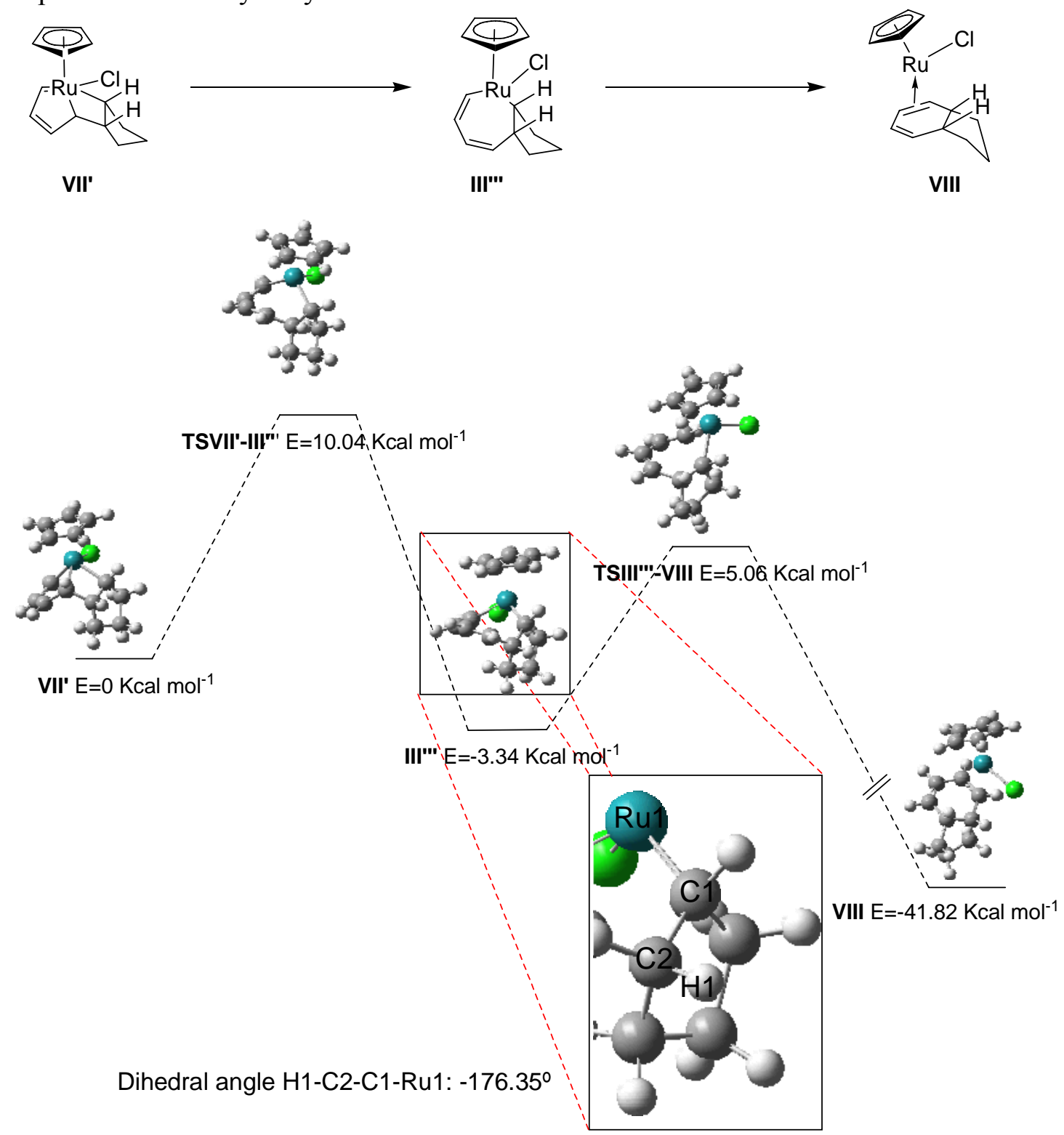

Scheme 3 
VI. Spectral data<smiles>COC(=O)C1=CC2=CC(C(=O)OC)(CC2)CC1C(=O)OC</smiles>

Trimethyl 6,7-dihydro-1H-indene-2,2,5(3H)-tricarboxylate (4a)

Pale yellow oil. ${ }^{1} \mathrm{H}-\mathrm{NMR}\left(400 \mathrm{MHz}, \mathrm{CDCl}_{3}\right), \delta(\mathrm{ppm}): 6.96(\mathrm{~s}, 1 \mathrm{H}), 3.75(\mathrm{~s}, 9 \mathrm{H}), 3.10(\mathrm{~s}, 4 \mathrm{H}), 2.55(\mathrm{t}$, $J=9.7 \mathrm{~Hz}, 2 \mathrm{H}), 2.27(\mathrm{t}, J=9.7 \mathrm{~Hz}, 2 \mathrm{H}) .{ }^{13} \mathrm{C}-\mathrm{NMR}$, DEPT $\left(100 \mathrm{MHz}, \mathrm{CDCl}_{3}\right), \delta(\mathrm{ppm}): 172.2(2 \times \mathrm{CO})$, $167.8(\mathrm{CO}), 141.1(\mathrm{C}), 131.7(\mathrm{CH}), 130.3(\mathrm{C}), 126.4(\mathrm{C}), 58.5(\mathrm{C}), 52.9\left(2 \times \mathrm{CH}_{3}\right), 51.3\left(\mathrm{CH}_{3}\right), 43.5$ $\left(\mathrm{CH}_{2}\right), 40.8\left(\mathrm{CH}_{2}\right), 23.4\left(\mathrm{CH}_{2}\right), 22.1\left(\mathrm{CH}_{2}\right) . \mathrm{MS}, \mathrm{m} / \mathrm{z}$ (\% relative intensity): $295\left(\mathrm{M}^{+}+1,96\right), 294(22)$, 293 (39), 263 (32), 235 (100). HRMS calculated for $\mathrm{C}_{15} \mathrm{H}_{19} \mathrm{O}_{6}: 295.1181$; found: 295.1175.

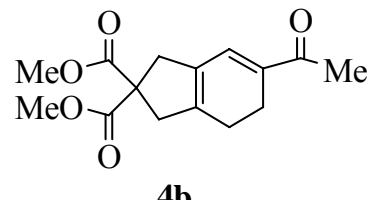

4b

Dimethyl 6-acetyl-4,5-dihydro-1H-indene-2,2(3H)-dicarboxylate (4b)

Pale yellow crystals, m.p. $83-84^{\circ} \mathrm{C}$ (hexane/ $\left.\mathrm{CH}_{2} \mathrm{Cl}_{2}\right) .{ }^{1} \mathrm{H}-\mathrm{NMR}\left(250 \mathrm{MHz}, \mathrm{CDCl}_{3}\right), \delta(\mathrm{ppm}): 6.78$ (s, 1H), $3.69(\mathrm{~s}, 6 \mathrm{H}), 3.09-3.05(\mathrm{~m}, 4 \mathrm{H}), 2.50-2.42(\mathrm{~m}, 4 \mathrm{H}), 2.42(\mathrm{~s}, 3 \mathrm{H}) .{ }^{13} \mathrm{C}-\mathrm{NMR}$, DEPT $(63 \mathrm{MHz}$, $\left.\mathrm{CDCl}_{3}\right), \delta(\mathrm{ppm}): 198.0(\mathrm{CO}), 172.1(2 \times \mathrm{CO}), 142.8(\mathrm{C}), 135.5(\mathrm{C}), 132.3(\mathrm{CH}), 130.3(\mathrm{C}), 58.4(\mathrm{C})$, $52.9\left(2 \times \mathrm{CH}_{3}\right), 43.4\left(\mathrm{CH}_{2}\right), 40.7\left(\mathrm{CH}_{2}\right), 25.0\left(\mathrm{CH}_{3}\right), 23.5\left(\mathrm{CH}_{2}\right), 20.7\left(\mathrm{CH}_{2}\right) . \mathrm{MS}, \mathrm{m} / \mathrm{z}(\%$ relative intensity): $279\left(\mathrm{M}^{+}+1,100\right), 278$ (15), 277 (35), 219 (76). HRMS calculated for $\mathrm{C}_{15} \mathrm{H}_{19} \mathrm{O}_{5}$ : 279.1232; found: 279.1234.

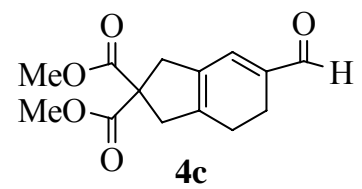

\section{Dimethyl 6-formyl-4,5-dihydro-1 $\mathrm{H}$-indene-2,2(3H)-dicarboxylate (4c)}

Pale yellow solid. ${ }^{1} \mathrm{H}-\mathrm{NMR}\left(250 \mathrm{MHz}, \mathrm{CDCl}_{3}\right), \delta(\mathrm{ppm}): 9.39(\mathrm{~s}, 1 \mathrm{H}), 6.64(\mathrm{~s}, 1 \mathrm{H}), 3.68(\mathrm{~s}, 6 \mathrm{H}), 3.11-$ $3.06(\mathrm{~m}, 4 \mathrm{H}), 2.48-2.40(\mathrm{~m}, 2 \mathrm{H}), 2.26-2.17(\mathrm{~m}, 2 \mathrm{H}) .{ }^{13} \mathrm{C}-\mathrm{NMR}$, DEPT $\left(63 \mathrm{MHz}, \mathrm{CDCl}_{3}\right), \delta(\mathrm{ppm})$ : $192.1(\mathrm{CHO}), 172.6(2 \times \mathrm{CO}), 145.9(\mathrm{C}), 140.5(\mathrm{CH}), 137.4(\mathrm{C}), 130.6(\mathrm{C}), 58.5(\mathrm{C}), 53.4\left(2 \times \mathrm{CH}_{3}\right), 43.8$ $\left(\mathrm{CH}_{2}\right), 40.7\left(\mathrm{CH}_{2}\right), 23.8\left(\mathrm{CH}_{2}\right), 19.3\left(\mathrm{CH}_{2}\right) . \mathrm{MS}, \mathrm{m} / \mathrm{z}$ (\% relative intensity): $265\left(\mathrm{M}^{+}+1,100\right), 264(10)$, 233 (19), 205 (72). HRMS calculated for $\mathrm{C}_{14} \mathrm{H}_{17} \mathrm{O}_{5}: 265.1076$; found: 265.1077.<smiles>COC(=O)C1(C(=O)OC)Cc2ccc(C=O)cc2C1</smiles>

\section{Dimethyl 5-formyl-1H-indene-2,2(3H)-dicarboxylate}

White solid. ${ }^{1} \mathrm{H}-\mathrm{NMR}\left(250 \mathrm{MHz}, \mathrm{CDCl}_{3}\right), \delta(\mathrm{ppm}): 9.86(\mathrm{~s}, 1 \mathrm{H}), 7.63-7.59(\mathrm{~m}, 2 \mathrm{H}), 7.26(\mathrm{~d}, J=7.7 \mathrm{~Hz}$, 1H), $3.67(\mathrm{~s}, 6 \mathrm{H}), 3.56(\mathrm{~s}, 4 \mathrm{H}) .{ }^{13} \mathrm{C}-\mathrm{NMR}, \mathrm{DEPT}\left(63 \mathrm{MHz}, \mathrm{CDCl}_{3}\right), \delta(\mathrm{ppm}): 192.0(\mathrm{CHO}), 171.7$ $(2 \times \mathrm{CO}), 147.3(\mathrm{C}), 141.1(\mathrm{C}), 136.0(\mathrm{C}), 129.7(\mathrm{CH}), 125.1(\mathrm{CH}), 124.8(\mathrm{CH}), 60.3(\mathrm{C}), 53.1\left(2 \times \mathrm{CH}_{3}\right)$, 
$40.7\left(\mathrm{CH}_{2}\right), 40.0\left(\mathrm{CH}_{2}\right) . \mathrm{MS}, \mathrm{m} / \mathrm{z}$ (\% relative intensity): $263\left(\mathrm{M}^{+}+1,100\right), 262(11), 231$ (46), $203(36)$. HRMS calculated for $\mathrm{C}_{14} \mathrm{H}_{15} \mathrm{O}_{5}: 263.0919$; found: 263.0921 .

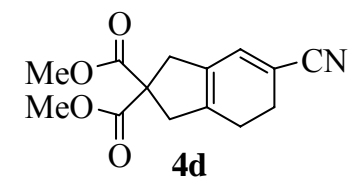

\section{Dimethyl 6-cyano-4,5-dihydro-1H-indene-2,2(3H)-dicarboxylate (4d)}

White crystals. m.p. $72-75^{\circ} \mathrm{C}$ (hexane/ ethyl acetate). ${ }^{1} \mathrm{H}-\mathrm{NMR}\left(500 \mathrm{MHz}, \mathrm{CDCl}_{3}\right), \delta(\mathrm{ppm}): 6.61$ (s, $1 \mathrm{H}), 3.75(\mathrm{~s}, 6 \mathrm{H}), 3.10(\mathrm{~s}, 4 \mathrm{H}), 2.74(\mathrm{t}, J=9.8 \mathrm{~Hz}, 2 \mathrm{H}), 2.31(\mathrm{t}, J=9.8 \mathrm{~Hz}, 2 \mathrm{H}) .{ }^{13} \mathrm{C}-\mathrm{NMR}$, DEPT $(126$ $\left.\mathrm{MHz}, \mathrm{CDCl}_{3}\right), \delta(\mathrm{ppm}): 172.0(2 \times \mathrm{CO}), 140.7(\mathrm{C}), 136.6(\mathrm{CH}), 129.5(\mathrm{C}), 119.7(\mathrm{C}), 115.7(\mathrm{CN}), 106.6$ (C), $58.1(\mathrm{C}), 53.1\left(2 \times \mathrm{CH}_{3}\right), 43.6\left(\mathrm{CH}_{2}\right), 40.7\left(\mathrm{CH}_{2}\right), 24.4\left(\mathrm{CH}_{2}\right), 22.7\left(\mathrm{CH}_{2}\right) . \mathrm{MS}, \mathrm{m} / \mathrm{z}(\%$ relative intensity): $262\left(\mathrm{M}^{+}+1,5\right), 41$ (46), 29 (100). HRMS calculated for $\mathrm{C}_{14} \mathrm{H}_{16} \mathrm{NO}_{4}$ : 262.1076; found: 262.1079 .

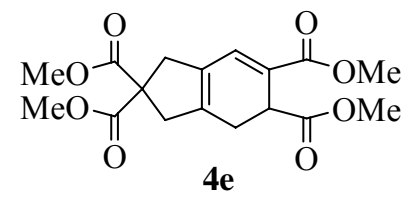

\section{Tetramethyl 4,5-dihydro-1 $\mathrm{H}$-indene-2,2,5,6(3H)-tetracarboxylate (4e)}

Pale yellow oil. ${ }^{1} \mathrm{H}-\mathrm{NMR}\left(300 \mathrm{MHz}, \mathrm{CDCl}_{3}\right), \delta(\mathrm{ppm}): 7.03(\mathrm{~s}, 1 \mathrm{H}), 3.71(\mathrm{~s}, 3 \mathrm{H}), 3.67(\mathrm{~s}, 6 \mathrm{H}), 3.59(\mathrm{~s}$, $3 \mathrm{H}), 3.14-2.77(\mathrm{~m}, 6 \mathrm{H}), 2.82-2.76(\mathrm{~m}, 1 \mathrm{H}) .{ }^{13} \mathrm{C}-\mathrm{NMR}$, DEPT $\left(75 \mathrm{MHz}, \mathrm{CDCl}_{3}\right), \delta(\mathrm{ppm}): 173.3(\mathrm{CO})$, $171.9(\mathrm{CO}), 171.6(\mathrm{CO}), 166.7(\mathrm{CO}), 140.1(\mathrm{C}), 132.4(\mathrm{CH}), 130.0(\mathrm{C}), 124.4(\mathrm{C}), 58.4(\mathrm{C}), 52.8$ $\left(\mathrm{CH}_{3}\right), 52.7\left(\mathrm{CH}_{3}\right), 52.2\left(\mathrm{CH}_{3}\right), 51.7\left(\mathrm{CH}_{3}\right), 43.0\left(\mathrm{CH}_{2}\right), 40.5\left(\mathrm{CH}_{2}\right), 38.1(\mathrm{CH}), 26.7\left(\mathrm{CH}_{2}\right) . \mathrm{MS}, \mathrm{m} / \mathrm{z}$ (\% relative intensity): $353\left(\mathrm{M}^{+}+1,27\right), 351$ (18), 321 (45), 319 (52), 293 (100), 261 (70). HRMS calculated for $\mathrm{C}_{17} \mathrm{H}_{21} \mathrm{O}_{8}$ : 353.1236 ; found: 353.1231 .

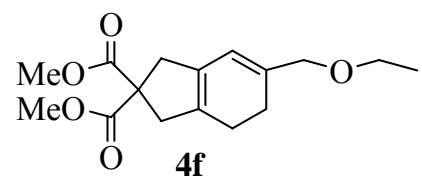

\section{Dimethyl 6-(ethoxymethyl)-4,5-dihydro-1H-indene-2,2(3H)-dicarboxylate (4f)}

Pale yellow oil. ${ }^{1} \mathrm{H}-\mathrm{NMR}\left(250 \mathrm{MHz}, \mathrm{CDCl}_{3}\right), \delta(\mathrm{ppm}): 5.76(\mathrm{~s}, 1 \mathrm{H}), 3.86(\mathrm{~s}, 2 \mathrm{H}), 3.67(\mathrm{~s}, 6 \mathrm{H}), 3.40(\mathrm{q}$, $J=7.0 \mathrm{~Hz}, 2 \mathrm{H}), 2.96(\mathrm{~s}, 4 \mathrm{H}), 2.16(\mathrm{~s}, 4 \mathrm{H}), 1.14(\mathrm{t}, J=7.0 \mathrm{~Hz}, 3 \mathrm{H}) .{ }^{13} \mathrm{C}-\mathrm{NMR}$, DEPT $\left(63 \mathrm{MHz}, \mathrm{CDCl}_{3}\right)$, $\delta(\mathrm{ppm}): 172.4(2 \times \mathrm{CO}), 135.3(\mathrm{C}), 131.9(\mathrm{C}), 129.9(\mathrm{C}), 119.2(\mathrm{CH}), 73.7\left(\mathrm{CH}_{2}\right), 65.3\left(\mathrm{CH}_{2}\right), 58.5(\mathrm{C})$, $52.8\left(2 \times \mathrm{CH}_{3}\right), 43.2\left(\mathrm{CH}_{2}\right), 41.4\left(\mathrm{CH}_{2}\right), 24.8\left(\mathrm{CH}_{2}\right), 23.2\left(\mathrm{CH}_{2}\right), 15.2\left(\mathrm{CH}_{3}\right) . \mathrm{MS}, \mathrm{m} / \mathrm{z}(\%$ relative intensity): 295 ( $\left.\mathrm{M}^{+}+1,31\right), 294$ (44), 293 (39), 249 (97), 247 (29), 236 (16), 235 (100), 189 (61). HRMS calculated for $\mathrm{C}_{16} \mathrm{H}_{23} \mathrm{O}_{5}$ : 295.1546; found: 295.1545 . 
<smiles>COC(=O)C1(C(=O)OC)CC2=C(CCC(COc3ccccc3)=C2)C1</smiles>

$4 \mathrm{~g}$

Dimethyl 4,5-dihydro-6-(phenoxymethyl)-1H-indene-2,2(3H)-dicarboxylate (4g)

Pale yelllow oil. ${ }^{1} \mathrm{H}-\mathrm{NMR}\left(250 \mathrm{MHz}, \mathrm{CDCl}_{3}\right), \delta(\mathrm{ppm})$ : 7.31-7.19 (m, 2H), 6.97-6.84 (m, 3H), $5.95(\mathrm{~s}$, 1H), $4.48(\mathrm{~s}, 2 \mathrm{H}), 3.74(\mathrm{~s}, 6 \mathrm{H}), 3.04(\mathrm{~s}, 4 \mathrm{H}), 2.37-2.24(\mathrm{~m}, 4 \mathrm{H}) .{ }^{13} \mathrm{C}-\mathrm{NMR}$, DEPT $\left(63 \mathrm{MHz}, \mathrm{CDCl}_{3}\right)$, $\delta(\mathrm{ppm}): 172.6(2 \times \mathrm{CO}), 158.7(\mathrm{C}), 133.4(\mathrm{C}), 132.4(\mathrm{C}), 129.3(2 \times \mathrm{CH}), 120.7(\mathrm{CH}), 119.8(\mathrm{CH}), 114.7$ $(2 \times \mathrm{CH}), 71.0\left(\mathrm{CH}_{2}\right), 58.4(\mathrm{C}), 52.8\left(2 \times \mathrm{CH}_{3}\right), 43.2\left(\mathrm{CH}_{2}\right), 41.3\left(\mathrm{CH}_{2}\right), 24.6\left(\mathrm{CH}_{2}\right), 23.1\left(\mathrm{CH}_{2}\right) . \mathrm{MS}, \mathrm{m} / \mathrm{z}$ (\% relative intensity): $343\left(\mathrm{M}^{+}+1,53\right), 342$ (39), 341 (55), 311 (32), 284 (36), 283 (90), 281 (39), 251 (38), 250 (60), 249 (100), 248 (41), 247 (77), 217 (31), 191 (65), 190 (52), 189 (90), 131 (31), 94 (61), 93 (31). HRMS calculated for $\mathrm{C}_{20} \mathrm{H}_{23} \mathrm{O}_{5}$ : 343.1545 ; found: 343.1538 .<smiles>COCC1=CC2=C(CC1)CC(C(C)=O)(C(C)=O)C2</smiles>

\section{Dimethyl 6-(trimethylsilyloximethyl)-2,3,4,5-tetrahydro-1H-2,2-indenedicarboxylate (4h)}

Pale yellow oil. ${ }^{1} \mathrm{H}-\mathrm{NMR}\left(250 \mathrm{MHz}, \mathrm{CDCl}_{3}\right), \delta(\mathrm{ppm}): 5.72(\mathrm{~s}, 1 \mathrm{H}), 3.98(\mathrm{~s}, 2 \mathrm{H}), 3.63(\mathrm{~s}, 6 \mathrm{H}), 2.92(\mathrm{~s}$, 4H), 2.10 (s, 4H), 0.03 (s, 9H). ${ }^{13} \mathrm{C}-\mathrm{NMR}, \mathrm{DEPT}\left(63 \mathrm{MHz}, \mathrm{CDCl}_{3}\right), \delta(\mathrm{ppm}): 172.7(2 \times \mathrm{CO}), 137.6(\mathrm{C})$, $131.1(\mathrm{C}), 130.0(\mathrm{C}), 116.9(\mathrm{CH}), 65.6\left(\mathrm{CH}_{2}\right), 58.5(\mathrm{C}), 52.8\left(2 \times \mathrm{CH}_{3}\right), 43.2\left(\mathrm{CH}_{2}\right), 41.4\left(\mathrm{CH}_{2}\right), 24.6$ $\left(\mathrm{CH}_{2}\right), 23.2\left(\mathrm{CH}_{2}\right),-0.42\left(3 \times \mathrm{CH}_{3}\right) . \mathrm{MS}, \mathrm{m} / \mathrm{z}$ (\% relative intensity): $323\left(\mathrm{M}^{+}-15,13\right), 279(16), 249(44)$, 247 (10), 217 (58), 190 (14), 189 (100), 91 (18), 75 (28), 73 (22).<smiles>COC(=O)C1(C(=O)OC)CC2=CC(CO)=CC2C1</smiles>

Dimethyl 4,5-dihydro-6-(hydroxymethyl)-1H-indene-2,2(3H)-dicarboxylate (4i)

Pale yellow oil. ${ }^{1} \mathrm{H}-\mathrm{NMR}\left(300 \mathrm{MHz}, \mathrm{CDCl}_{3}\right), \delta(\mathrm{ppm}): 5.83(\mathrm{~s}, 1 \mathrm{H}), 4.09-4.08(\mathrm{~m}, 2 \mathrm{H}), 3.73(\mathrm{~s}, 3 \mathrm{H})$, $3.72(\mathrm{~s}, 3 \mathrm{H}), 3.02(\mathrm{~s}, 4 \mathrm{H}), 2.24-2.20(\mathrm{~m}, 4 \mathrm{H}), 2.03(\mathrm{~s}, 1 \mathrm{H}),{ }^{13} \mathrm{C}-\mathrm{NMR}$, DEPT $\left(75 \mathrm{MHz}, \mathrm{CDCl}_{3}\right), \delta$ (ppm): $172.7(2 \times \mathrm{CO}), 137.5(\mathrm{C}), 131.8(\mathrm{C}), 129.9(\mathrm{C}), 117.5(\mathrm{CH}), 66.1\left(\mathrm{CH}_{2}\right), 58.6(\mathrm{C}), 52.9$ $\left(2 \times \mathrm{CH}_{3}\right), 43.1\left(\mathrm{CH}_{2}\right), 41.3\left(\mathrm{CH}_{2}\right), 24.6\left(\mathrm{CH}_{2}\right), 23.3\left(\mathrm{CH}_{2}\right) . \mathrm{MS}, \mathrm{m} / \mathrm{z}$ (\% relative intensity): $267\left(\mathrm{M}^{+}+1\right.$, 2), 249 (46), 217 (72), 191 (23), 189 (100).

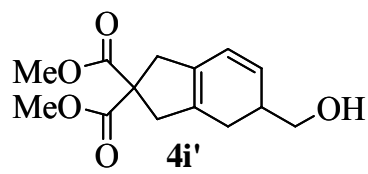

Dimethyl 4,5-dihydro-5-(hydroxymethyl)-1H-indene-2,2(3H)-dicarboxylate (4i') 
Pale yellow oil. ${ }^{1} \mathrm{H}-\mathrm{NMR}\left(300 \mathrm{MHz}, \mathrm{CDCl}_{3}\right), \delta(\mathrm{ppm}): 5.51(\mathrm{~s}, 1 \mathrm{H}), 5.46(\mathrm{~s}, 1 \mathrm{H}), 3.71(\mathrm{~s}, 3 \mathrm{H}), 3.70(\mathrm{~s}$, $3 \mathrm{H}), 3.54-3.50(\mathrm{~m}, 2 \mathrm{H}), 2.93-2.90(\mathrm{~m}, 4 \mathrm{H}), 2.03(\mathrm{~s}, 1 \mathrm{H}), 1.85-1.66(\mathrm{~m}, 3 \mathrm{H}) .{ }^{13} \mathrm{C}-\mathrm{NMR}$, DEPT $(75$ $\left.\mathrm{MHz}, \mathrm{CDCl}_{3}\right), \delta(\mathrm{ppm}): 171.7(2 \times \mathrm{CO}), 126.0(\mathrm{C}), 123.0(\mathrm{C}), 117.6(\mathrm{CH}), 116.7(\mathrm{CH}), 66.0\left(\mathrm{CH}_{2}\right), 58.5$ (C), $52.9\left(2 \times \mathrm{CH}_{3}\right), 38.8\left(\mathrm{CH}_{2}\right), 38.4\left(\mathrm{CH}_{2}\right), 36.2(\mathrm{CH}), 26.0\left(\mathrm{CH}_{2}\right) . \mathrm{MS}, \mathrm{m} / \mathrm{z}$ (\% relative intensity): 267 $\left(\mathrm{M}^{+}+1,2\right), 249$ (33), 217 (46), 207 (37), 189 (100), 57 (59).

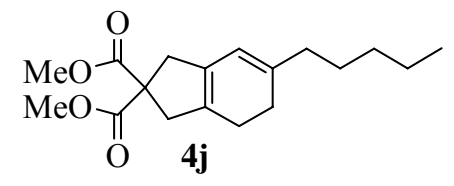

Dimethyl 4,5-dihydro-6-pentyl-1H-indene-2,2(3H)-dicarboxylate (4j)

Pale yellow oil. ${ }^{1} \mathrm{H}-\mathrm{NMR}\left(400 \mathrm{MHz}, \mathrm{CDCl}_{3}\right), \delta(\mathrm{ppm}): 5.58(\mathrm{~s}, 1 \mathrm{H}), 3.73(\mathrm{~s}, 6 \mathrm{H}), 3.01(\mathrm{~s}, 4 \mathrm{H}), 2.17(\mathrm{~s}$, 4H), $2.04(\mathrm{t}, J=7.5 \mathrm{~Hz}, 2 \mathrm{H}), 1.46-1.38(\mathrm{~m}, 2 \mathrm{H}), 1.32-1.26(\mathrm{~m}, 4 \mathrm{H}), 0.89$ (t, $J=7.1 \mathrm{~Hz}, 3 \mathrm{H}) .{ }^{13} \mathrm{C}-\mathrm{NMR}$, DEPT $\left(100 \mathrm{MHz}, \mathrm{CDCl}_{3}\right), \delta(\mathrm{ppm}): 172.8(2 \times \mathrm{CO}), 139.7(\mathrm{C}), 130.7(\mathrm{C}), 129.2(\mathrm{C}), 116.6(\mathrm{CH}), 58.5$ (C), $52.7\left(2 \times \mathrm{CH}_{3}\right), 43.2\left(\mathrm{CH}_{2}\right), 41.6\left(\mathrm{CH}_{2}\right), 37.1\left(\mathrm{CH}_{2}\right), 31.7\left(\mathrm{CH}_{2}\right), 27.6\left(\mathrm{CH}_{2}\right), 27.4\left(\mathrm{CH}_{2}\right), 23.6$ $\left(\mathrm{CH}_{2}\right), 22.5\left(\mathrm{CH}_{2}\right), 14.1\left(\mathrm{CH}_{3}\right) . \mathrm{MS}, \mathrm{m} / \mathrm{z}$ (\% relative intensity): $307\left(\mathrm{M}^{+}+1,12\right), 306(21), 305(20), 247$ (32). HRMS calculated for $\mathrm{C}_{18} \mathrm{H}_{27} \mathrm{O}_{4}$ : 307.1909; found: 307.1901 .

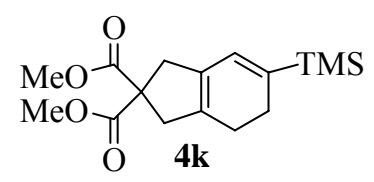

Dimethyl 4,5-dihydro-6-(trimethylsilyl)-1H-indene-2,2(3H)-dicarboxylate (4k)

Colorless oil. ${ }^{1} \mathrm{H}-\mathrm{NMR}\left(400 \mathrm{MHz}, \mathrm{CDCl}_{3}\right), \delta(\mathrm{ppm}): 6.10(\mathrm{~s}, 1 \mathrm{H}), 3.72(\mathrm{~s}, 6 \mathrm{H}), 3.05-3.03(\mathrm{~m}, 4 \mathrm{H}), 2.28$ $(\mathrm{t}, J=9.5 \mathrm{~Hz}, 2 \mathrm{H}), 2.08(\mathrm{t}, J=9.6 \mathrm{~Hz}, 2 \mathrm{H}), 0.06(\mathrm{~s}, 9 \mathrm{H}) .{ }^{13} \mathrm{C}-\mathrm{NMR}$, DEPT $\left(100 \mathrm{MHz}, \mathrm{CDCl}_{3}\right), \delta(\mathrm{ppm})$ : $172.7(2 \times \mathrm{CO}), 138.5(\mathrm{C}), 134.1(\mathrm{C}), 130.3(\mathrm{C}), 129.9(\mathrm{CH}), 58.2(\mathrm{C}), 52.7\left(2 \times \mathrm{CH}_{3}\right), 43.5\left(\mathrm{CH}_{2}\right), 41.4$ $\left(\mathrm{CH}_{2}\right), 25.3\left(\mathrm{CH}_{2}\right), 23.1\left(\mathrm{CH}_{2}\right),-2.20\left(3 \times \mathrm{CH}_{3}\right) . \mathrm{MS}, \mathrm{m} / \mathrm{z}$ (\% relative intensity): $309\left(\mathrm{M}^{+}+1,100\right), 308$ (90), 307 (68), 293 (45), 291 (27), 275 (23), 249 (83), 248 (20), 247 (36), 203 (26), 177 (22), 175 (36), 113 (20), 73 (26). HRMS calculated for $\mathrm{C}_{16} \mathrm{H}_{25} \mathrm{O}_{4} \mathrm{Si}$ : 309.1522 ; found: 309.1518 .

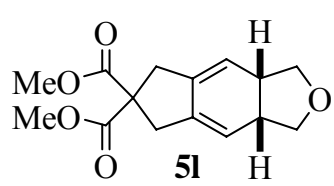

Dimethyl 3,3a,5,6,7,8a-hexahydro-1H-cyclopenta[f]isobenzofuran-6,6-dicarboxylate (5I)

Yellowish solid, m.p. 67-68 ${ }^{\circ} \mathrm{C}$ (hexane/ $\left.\mathrm{CH}_{2} \mathrm{Cl}_{2}\right)$ [lit. ${ }^{4}$ 51-52 $\left.{ }^{\circ} \mathrm{C}\left(\mathrm{CHCl}_{3}\right)\right] .{ }^{1} \mathrm{H}-\mathrm{NMR}(300 \mathrm{MHz}$, $\left.\mathrm{CDCl}_{3}\right), \delta(\mathrm{ppm}): 5.36(\mathrm{~s}, 2 \mathrm{H}), 4.09-4.04(\mathrm{~m}, 2 \mathrm{H}), 3.66(\mathrm{~s}, 3 \mathrm{H}), 3.65(\mathrm{~s}, 3 \mathrm{H}), 3.45-3.40(\mathrm{~m}, 2 \mathrm{H}), 2.97-$ $2.81(\mathrm{~m}, 6 \mathrm{H}) .{ }^{13} \mathrm{C}-\mathrm{NMR}$, DEPT $\left(75 \mathrm{MHz}, \mathrm{CDCl}_{3}\right), \delta(\mathrm{ppm}): 171.6(2 \times \mathrm{CO}), 134.8(2 \times \mathrm{C}), 117.3$ $(2 \times \mathrm{CH}), 75.1\left(2 \times \mathrm{CH}_{2}\right), 58.6(\mathrm{C}), 52.6\left(2 \times \mathrm{CH}_{3}\right), 38.7\left(2 \times \mathrm{CH}_{2}\right), 38.6(2 \times \mathrm{CH}) . \mathrm{MS}, \mathrm{m} / \mathrm{z}(\%$ relative intensity): $279\left(\mathrm{M}^{+}+1,65\right), 278$ (25), 261 (38), 247 (28), 229 (16), 220 (21), 219 (100), 201 (38), 129 (16). HRMS calculated for $\mathrm{C}_{15} \mathrm{H}_{19} \mathrm{O}_{5}: 279.1232$; found: 279.1239 . 


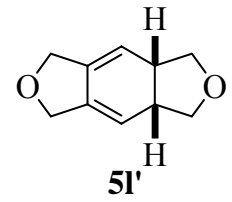

3a,5,7,8a-Tetrahydro-1H,3H-furo[3,4-f]isobenzofuran (5l')

Yellowish solid, m.p. $116-118^{\circ} \mathrm{C}$ (hexane/ ethyl acetate). ${ }^{1} \mathrm{H}-\mathrm{NMR}\left(300 \mathrm{MHz}, \mathrm{CDCl}_{3}\right), \delta$ (ppm): 5.36 $(\mathrm{s}, 2 \mathrm{H}), 4.41-4.29(\mathrm{~m}, 4 \mathrm{H}), 4.14-4.09(\mathrm{~m}, 2 \mathrm{H}), 3.50-3.45(\mathrm{~m}, 2 \mathrm{H}), 2.99(\mathrm{~s}, 2 \mathrm{H}) .{ }^{13} \mathrm{C}-\mathrm{NMR}$, DEPT $(75$ $\left.\mathrm{MHz}, \mathrm{CDCl}_{3}\right), \delta(\mathrm{ppm}): 135.0(2 \times \mathrm{C}), 114.0(2 \times \mathrm{CH}), 75.1\left(2 \times \mathrm{CH}_{2}\right), 70.3\left(2 \times \mathrm{CH}_{2}\right), 39.0(2 \times \mathrm{CH}) . \mathrm{MS}$, $\mathrm{m} / \mathrm{z}$ (\% relative intensity): $165\left(\mathrm{M}^{+}+1,29\right), 163(38), 147$ (60), 135 (64), 133 (28), 119 (100). HRMS calculated for $\mathrm{C}_{10} \mathrm{H}_{13} \mathrm{O}_{2}$ : 165.0915; found: 165.0910 .

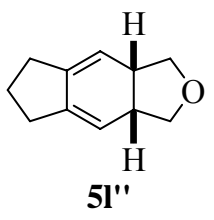

3,3a,5,6,7,8a-Hexahydro-1H-cyclopenta[f]isobenzofuran (5l’')

Pale yellow oil. ${ }^{1} \mathrm{H}-\mathrm{NMR}\left(400 \mathrm{MHz}, \mathrm{CDCl}_{3}\right), \delta(\mathrm{ppm}): 5.34-5.33(\mathrm{~m}, 2 \mathrm{H}), 4.12-4.08(\mathrm{~m}, 2 \mathrm{H}), 3.52-$ $3.48(\mathrm{~m}, 2 \mathrm{H}), 2.95-2.94(\mathrm{~m}, 2 \mathrm{H}), 2.36-2.22(\mathrm{~m}, 4 \mathrm{H}), 1.72-1.60(\mathrm{~m}, 2 \mathrm{H}) .{ }^{13} \mathrm{C}-\mathrm{NMR}$, DEPT $(100 \mathrm{MHz}$, $\left.\mathrm{CDCl}_{3}\right), \delta(\mathrm{ppm}): 138.7(2 \times \mathrm{C}), 115.7(2 \times \mathrm{CH}), 75.4\left(2 \times \mathrm{CH}_{2}\right), 38.9(2 \times \mathrm{CH}), 31.2\left(2 \times \mathrm{CH}_{2}\right), 24.7\left(\mathrm{CH}_{2}\right)$. MS, m/z (\% relative intensity): $163\left(\mathrm{M}^{+}+1,33\right), 161$ (100), 159 (29), 149 (25), 147 (53), 133 (53), 131 (25).

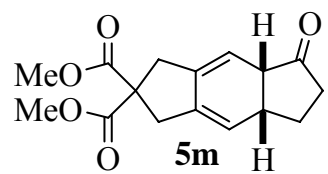

Dimethyl 5,6,7,7a-tetrahydro-5-oxo $s$-indacene-2,2(1H,3H,4aH)-dicarboxylate $(5 \mathrm{~m}) *$

Pale yellow oil. ${ }^{1} \mathrm{H}-\mathrm{NMR}\left(300 \mathrm{MHz}, \mathrm{CDCl}_{3}\right), \delta(\mathrm{ppm}): 5.60(\mathrm{~s}, 1 \mathrm{H}), 5.43(\mathrm{~s}, 1 \mathrm{H}), 3.73(\mathrm{~s}, 3 \mathrm{H}), 3.72(\mathrm{~s}$, $3 \mathrm{H}), 3.05-2.89(\mathrm{~m}, 6 \mathrm{H}), 2.26-2.04(\mathrm{~m}, 3 \mathrm{H}), 1.84-1.74(\mathrm{~m}, 1 \mathrm{H}) .{ }^{13} \mathrm{C}-\mathrm{NMR}$, DEPT $\left(75 \mathrm{MHz}, \mathrm{CDCl}_{3}\right), \delta$ (ppm): $219.8(\mathrm{CO}), 171.5(\mathrm{CO}), 171.4(\mathrm{CO}), 136.2(\mathrm{C}), 135.3(\mathrm{C}), 119.9(\mathrm{CH}), 112.4(\mathrm{CH}), 58.5(\mathrm{C})$, $52.8\left(2 \times \mathrm{CH}_{3}\right), 48.8(\mathrm{CH}), 38.6\left(2 \times \mathrm{CH}_{2}\right), 36.4(\mathrm{CH}), 36.2\left(\mathrm{CH}_{2}\right), 29.2\left(\mathrm{CH}_{2}\right) . \mathrm{MS}, \mathrm{m} / \mathrm{z}(\%$ relative intensity): $291\left(\mathrm{M}^{+}+1,16\right), 290$ (14), 289 (35), 231 (100). HRMS calculated for $\mathrm{C}_{16} \mathrm{H}_{19} \mathrm{O}_{5}$ : 291.1232; found: 291.1233 .

*Note: reaction performed using acetone as solvent.<smiles>COC(=O)C1(C(=O)OC)Cc2cc3c(cc2C1)C(=O)CC3</smiles>

Dimethyl 5,6-dihydro-7-oxo s-indacene-2,2(1H,3H,5H)-dicarboxylate

White crystals, m.p. $90-93^{\circ} \mathrm{C}$ (hexane/ ethyl acetate). ${ }^{1} \mathrm{H}-\mathrm{NMR}\left(300 \mathrm{MHz}, \mathrm{CDCl}_{3}\right), \delta$ (ppm): $7.53(\mathrm{~s}$, 1H), $7.27(\mathrm{~s}, 1 \mathrm{H}), 3.73(\mathrm{~s}, 6 \mathrm{H}), 3.59(\mathrm{~s}, 2 \mathrm{H}), 3.57(\mathrm{~s}, 2 \mathrm{H}), 3.05(\mathrm{t}, J=5.8 \mathrm{~Hz}, 2 \mathrm{H}), 2.65(\mathrm{t}, J=5.8 \mathrm{~Hz}$, 2H). ${ }^{13} \mathrm{C}-\mathrm{NMR}$, DEPT $\left(75 \mathrm{MHz}, \mathrm{CDCl}_{3}\right), \delta(\mathrm{ppm}): 206.4(\mathrm{CO}), 171.8(2 \times \mathrm{CO}), 155.0(\mathrm{C}), 148.3(\mathrm{C})$, $139.7(\mathrm{C}), 136.6(\mathrm{C}), 122.3(\mathrm{CH}), 119.2(\mathrm{CH}), 60.6(\mathrm{C}), 53.0\left(2 \times \mathrm{CH}_{3}\right), 40.4\left(\mathrm{CH}_{2}\right), 39.6\left(\mathrm{CH}_{2}\right), 36.6$ $\left(\mathrm{CH}_{2}\right), 25.5\left(\mathrm{CH}_{2}\right) . \mathrm{MS}, \mathrm{m} / \mathrm{z}$ (\% relative intensity): $289\left(\mathrm{M}^{+}+1,100\right), 288$ (7), 257 (19), 229 (21). HRMS calculated for $\mathrm{C}_{16} \mathrm{H}_{17} \mathrm{O}_{5}$ : 289.1076; found: 289.1073 . 
<smiles>COC(=O)C1(C(=O)OC)Cc2cc3c(cc2C1)C(=O)C=CC3=O</smiles>

Dimethyl 5,8-dioxo-1H-cyclopenta[b]naphthalene-2,2(3H,5H,8H)-dicarboxylate (5n)

Orangish solid. M. p. $115-117^{\circ} \mathrm{C}$ (hexane/ $\left.\mathrm{Cl}_{2} \mathrm{CH}_{2}\right) .{ }^{1} \mathrm{H}-\mathrm{NMR}\left(400 \mathrm{MHz}, \mathrm{CDCl}_{3}\right), \delta$ (ppm): $7.92(\mathrm{~s}, 2 \mathrm{H})$, $6.93(\mathrm{~s}, 2 \mathrm{H}), 3.77(\mathrm{~s}, 6 \mathrm{H}), 3.70(\mathrm{~s}, 4 \mathrm{H}) .{ }^{13} \mathrm{C}-\mathrm{NMR}$, DEPT $\left(100 \mathrm{MHz}, \mathrm{CDCl}_{3}\right), \delta(\mathrm{ppm}): 184.9(2 \times \mathrm{CO})$, $171.2(2 \times \mathrm{CO}), 146.6(2 \times \mathrm{C}), 138.7(2 \times \mathrm{CH}), 131.8(2 \times \mathrm{C}), 122.8(2 \times \mathrm{CH}), 60.0(\mathrm{C}), 53.2\left(2 \times \mathrm{CH}_{2}\right), 40.8$ $\left(2 \times \mathrm{CH}_{3}\right) . \mathrm{MS}, \mathrm{m} / \mathrm{z}$ (\% relative intensity): $315\left(\mathrm{M}^{+}+1,100\right), 283$ (6), 255 (7), 85 (7), 83 (11), 57 (6). HRMS calculated for $\mathrm{C}_{17} \mathrm{H}_{15} \mathrm{O}_{6}: 315.0869$; found: 315.0870 .

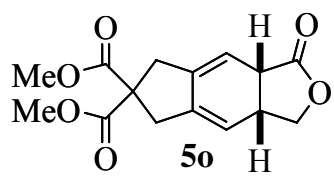

Dimethyl 1-oxo-3,3a,5,6,7,8a-hexahydro-1H-cyclopenta[f]isobenzofuran-6,6-dicarboxytate (5o) Colorless oil. ${ }^{1} \mathrm{H}-\mathrm{NMR}\left(300 \mathrm{MHz}, \mathrm{CDCl}_{3}\right), \delta(\mathrm{ppm})$ : 5.48 (s, 2H), 4.48-4.42 (m, 1H), 3.95-3.89 (m, $1 \mathrm{H}), 3.67(\mathrm{~s}, 3 \mathrm{H}), 3.66(\mathrm{~s}, 3 \mathrm{H}), 3.32(\mathrm{~s}, 2 \mathrm{H}), 3.04-2.84(\mathrm{~m}, 4 \mathrm{H}) .{ }^{13} \mathrm{C}-\mathrm{NMR}$, DEPT $\left(75 \mathrm{MHz}, \mathrm{CDCl}_{3}\right), \delta$ (ppm): $178.0(\mathrm{CO}), 171.2(\mathrm{CO}), 171.1(\mathrm{CO}), 137.3(\mathrm{C}), 137.2(\mathrm{C}), 115.3(\mathrm{CH}), 110.7(\mathrm{CH}), 73.6\left(\mathrm{CH}_{2}\right)$, $58.2(\mathrm{C}), 52.8\left(2 \times \mathrm{CH}_{3}\right), 40.6(\mathrm{CH}), 38.5\left(2 \times \mathrm{CH}_{2}\right), 35.4(\mathrm{CH}) . \mathrm{MS}, \mathrm{m} / \mathrm{z}$ (\% relative intensity): 293 $\left(\mathrm{M}^{+}+1,45\right), 291(12), 261(20), 233(100)$. HRMS calculated for $\mathrm{C}_{15} \mathrm{H}_{17} \mathrm{O}_{6}: 293.1025$; found: 293.1026 .

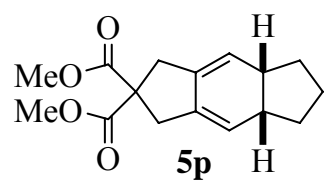

Dimethyl 4a,5,6,7-tetrahydro s-indacene-2,2(1H,3H,4aH)-dicarboxylate (5p)

Pale yellow oil. ${ }^{1} \mathrm{H}-\mathrm{NMR}\left(400 \mathrm{MHz}, \mathrm{CDCl}_{3}\right), \delta(\mathrm{ppm}): 5.37(\mathrm{~s}, 2 \mathrm{H}), 3.68(\mathrm{~s}, 3 \mathrm{H}), 3.67(\mathrm{~s}, 3 \mathrm{H}), 2.87(\mathrm{q}$, $J=16.2 \mathrm{~Hz}, 4 \mathrm{H}), 2.61(\mathrm{~s}, 2 \mathrm{H}), 1.97-1.87(\mathrm{~m}, 2 \mathrm{H}), 1.43-1.21(\mathrm{~m}, 4 \mathrm{H}) .{ }^{13} \mathrm{C}-\mathrm{NMR}$, DEPT $(100 \mathrm{MHz}$, $\left.\mathrm{CDCl}_{3}\right), \delta(\mathrm{ppm}): 171.8(2 \times \mathrm{CO}), 132.5(2 \times \mathrm{C}), 121.6(2 \times \mathrm{CH}), 59.1(\mathrm{C}), 53.3\left(2 \times \mathrm{CH}_{3}\right), 38.8\left(2 \times \mathrm{CH}_{2}\right)$, $37.9(2 \times \mathrm{CH}), 35.2\left(2 \times \mathrm{CH}_{2}\right), 23,2\left(\mathrm{CH}_{2}\right) . \mathrm{MS}, \mathrm{m} / \mathrm{z}$ (\% relative intensity): $277\left(\mathrm{M}^{+}+1,4\right), 41(100), 28$ (74), 27 (84). HRMS calculated for $\mathrm{C}_{16} \mathrm{H}_{21} \mathrm{O}_{4}: 277.1440$; found: 277.1448.

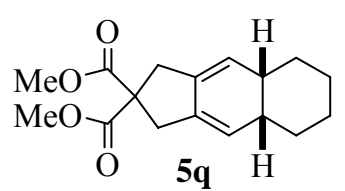

Dimethyl 4a,5,6,7,8,8a-hexahydro-1H-cyclopenta[b]naphthalene- 2,2(3H)- dicarboxylate (5q) Pale yellow oil. ${ }^{1} \mathrm{H}-\mathrm{NMR}\left(400 \mathrm{MHz}, \mathrm{CDCl}_{3}\right), \delta(\mathrm{ppm}): 5.43(\mathrm{~s}, 2 \mathrm{H}), 3.72(\mathrm{~s}, 3 \mathrm{H}), 3.71(\mathrm{~s}, 3 \mathrm{H}), 2.94(\mathrm{~s}$, 4H), $2.30(\mathrm{~s}, 2 \mathrm{H}), 1.43-1.41(\mathrm{~m}, 4 \mathrm{H}), 1.30-1.24(\mathrm{~m}, 4 \mathrm{H}) .{ }^{13} \mathrm{C}-\mathrm{NMR}$, DEPT $\left(100 \mathrm{MHz}, \mathrm{CDCl}_{3}\right), \delta$ (ppm): $171.9(2 \times \mathrm{CO}), 135.9(2 \times \mathrm{C}), 122.9(2 \times \mathrm{CH}), 59.3(\mathrm{C}), 52.7\left(\mathrm{CH}_{3}\right), 52.6\left(\mathrm{CH}_{3}\right), 38.5\left(2 \times \mathrm{CH}_{2}\right)$, $35.8(2 \times \mathrm{CH}), 28.3\left(2 \times \mathrm{CH}_{2}\right), 23.4\left(2 \times \mathrm{CH}_{2}\right) . \mathrm{MS}, \mathrm{m} / \mathrm{z}\left(\%\right.$ relative intensity): $291\left(\mathrm{M}^{+}+1,4\right), 289(20), 59$ (18), 41 (100), 29 (86). HRMS calculated for $\mathrm{C}_{17} \mathrm{H}_{22} \mathrm{O}_{4}$ : 291.1587; found: 291.1582. 
(1) Trost, B. M.; Toste, F. D. J. Am. Chem. Soc. 2002, 124, 5025.

(2) Trost, B. M.; Older, C. M. Organometallics 2002, 21, 2544.

(3) Schrenk, J. L.; McNair, A. M.; McCormick, F. B. Mann, K. R. Inorg. Chem. 1986, 25, 3501.

(4) M. J. Frisch, G. W. Trucks, H. B. Schlegel, G. E. Scuseria, M. A. Robb, J. R. Cheeseman, J. A. Montgomery, Jr., T. Vreven, K. N. Kudin, J. C. Burant, J. M. Millam, S. S. Iyengar, J. Tomasi, V. Barone, B. Mennucci, M. Cossi, G. Scalmani, N. Rega, G. A. Petersson, H. Nakatsuji, M. Hada, M. Ehara, K. Toyota, R. Fukuda, J. Hasegawa, M. Ishida, T. Nakajima, Y. Honda, O. Kitao, H. Nakai, M. Klene, X. Li, J. E. Knox, H. P. Hratchian, J. B. Cross, C. Adamo, J. Jaramillo, R. Gomperts, R. E. Stratmann, O. Yazyev, A. J. Austin, R. Cammi, C. Pomelli, J. W. Ochterski, P. Y. Ayala, K. Morokuma, G. A. Voth, P. Salvador, J. J. Dannenberg, V. G. Zakrzewski, S. Dapprich, A. D. Daniels, M. C. Strain, O. Farkas, D. K. Malick, A. D. Rabuck, K. Raghavachari, J. B. Foresman, J. V. Ortiz, Q. Cui, A. G. Baboul, S. Clifford, J. Cioslowski, B. B. Stefanov, G. Liu, A. Liashenko, P. Piskorz, I. Komaromi, R. L. Martin, D. J. Fox, T. Keith, M. A. Al-Laham, C. Y. Peng, A. Nanayakkara, M. Challacombe, P. M. W. Gill, B. Johnson, W. Chen, M. W. Wong, C. Gonzalez, and J. A. Pople, Gaussian 03, Revision C.01, Gaussian, Inc., Wallingford CT, 2004.

(5) (a) Stephens, P.J.; Devlin, F.J.; Chabalowski, C.F.; Frisch, M.J. J. Phys. Chem 1994, 98, 11623. (b) Kohn, W.; Becke, A.D.; Parr, R.G. J. Phys. Chem., 1996, 100, 12974.

(6) (a) T. H. Dunning Jr. and P. J. Hay, in Modern Theoretical Chemistry, Ed. H. F. Schaefer III, Vol. 3 (Plenum, New York, 1976) 1-28. (b) P. J. Hay and W. R. Wadt, J. Chem. Phys. 1985, 82, 270. (c) W. R. Wadt and P. J. Hay, J. Chem. Phys. 1985, 82, 284. (d) P. J. Hay and W. R. Wadt, J. Chem. Phys. 1985, 82, 299. 
VIII. Spectra<smiles>COC(=O)C1=CC2=C(CC1)CC(C(=O)OC)(C(=O)OC)C2</smiles>
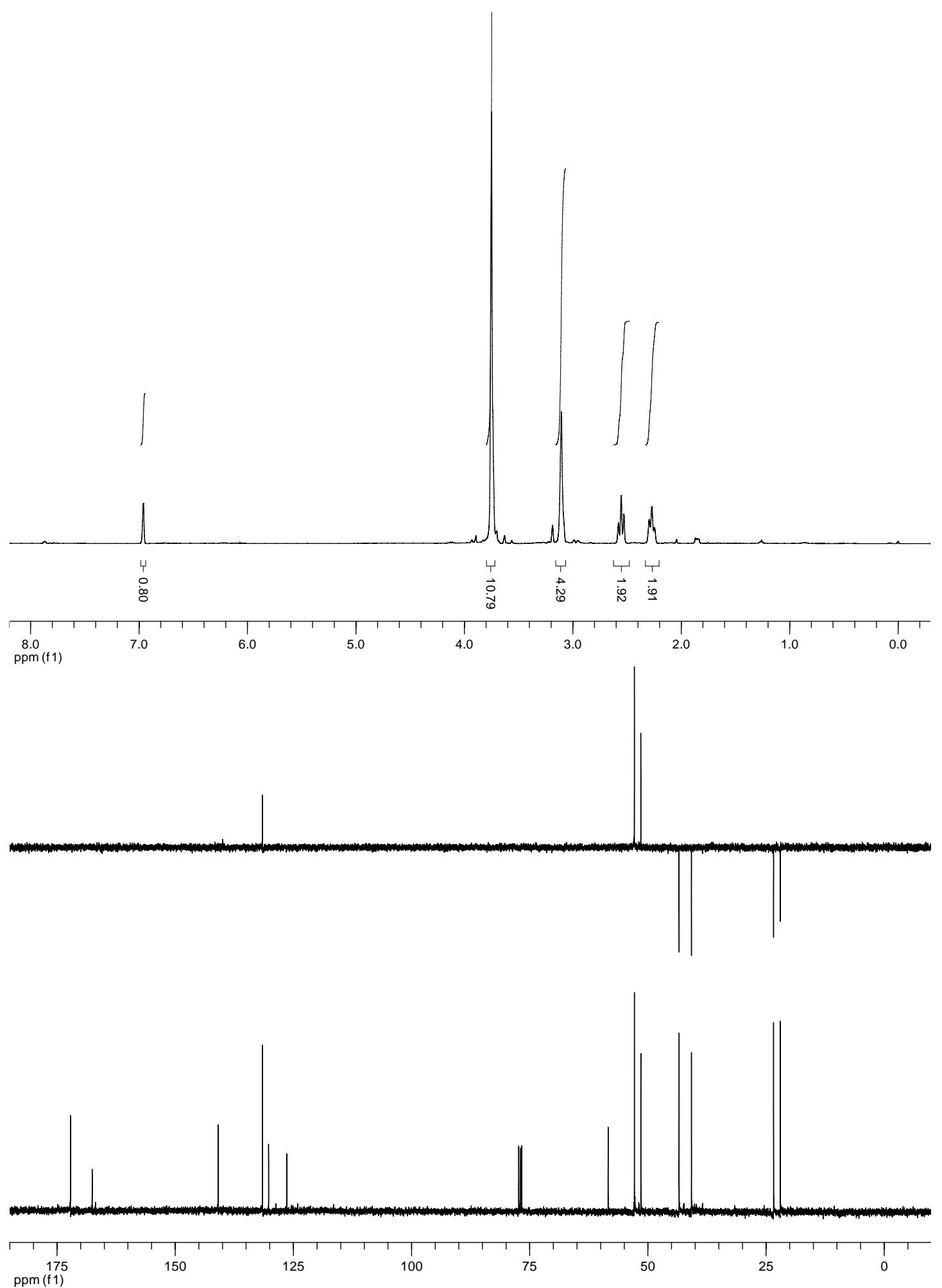


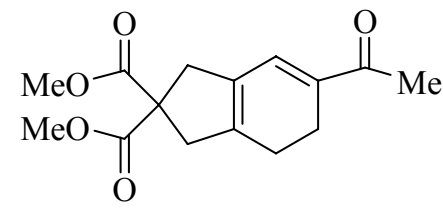

4b
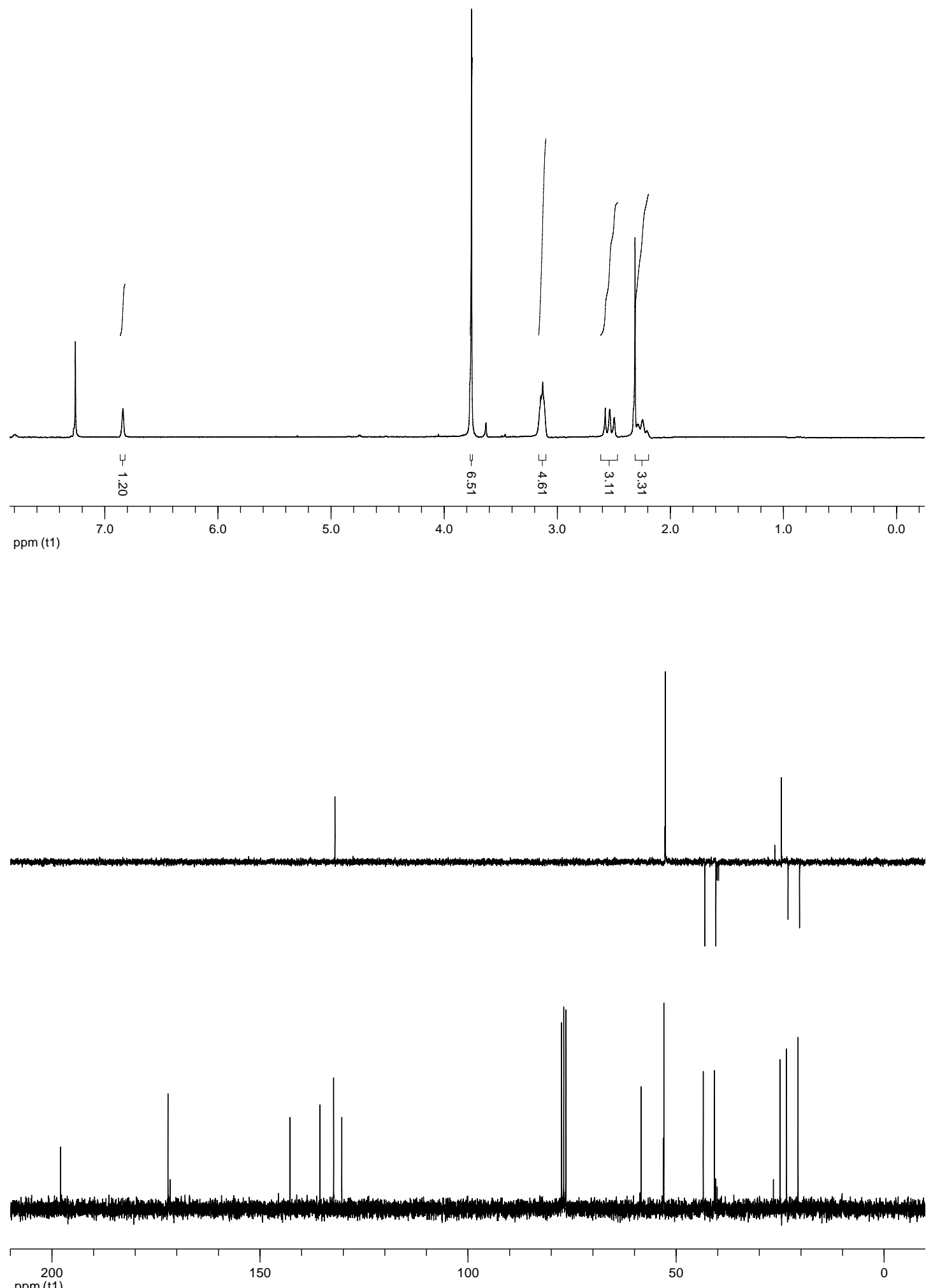


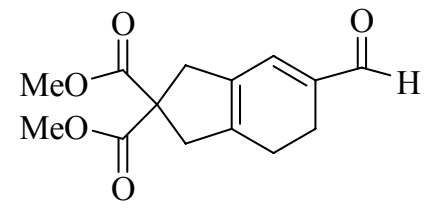

4c
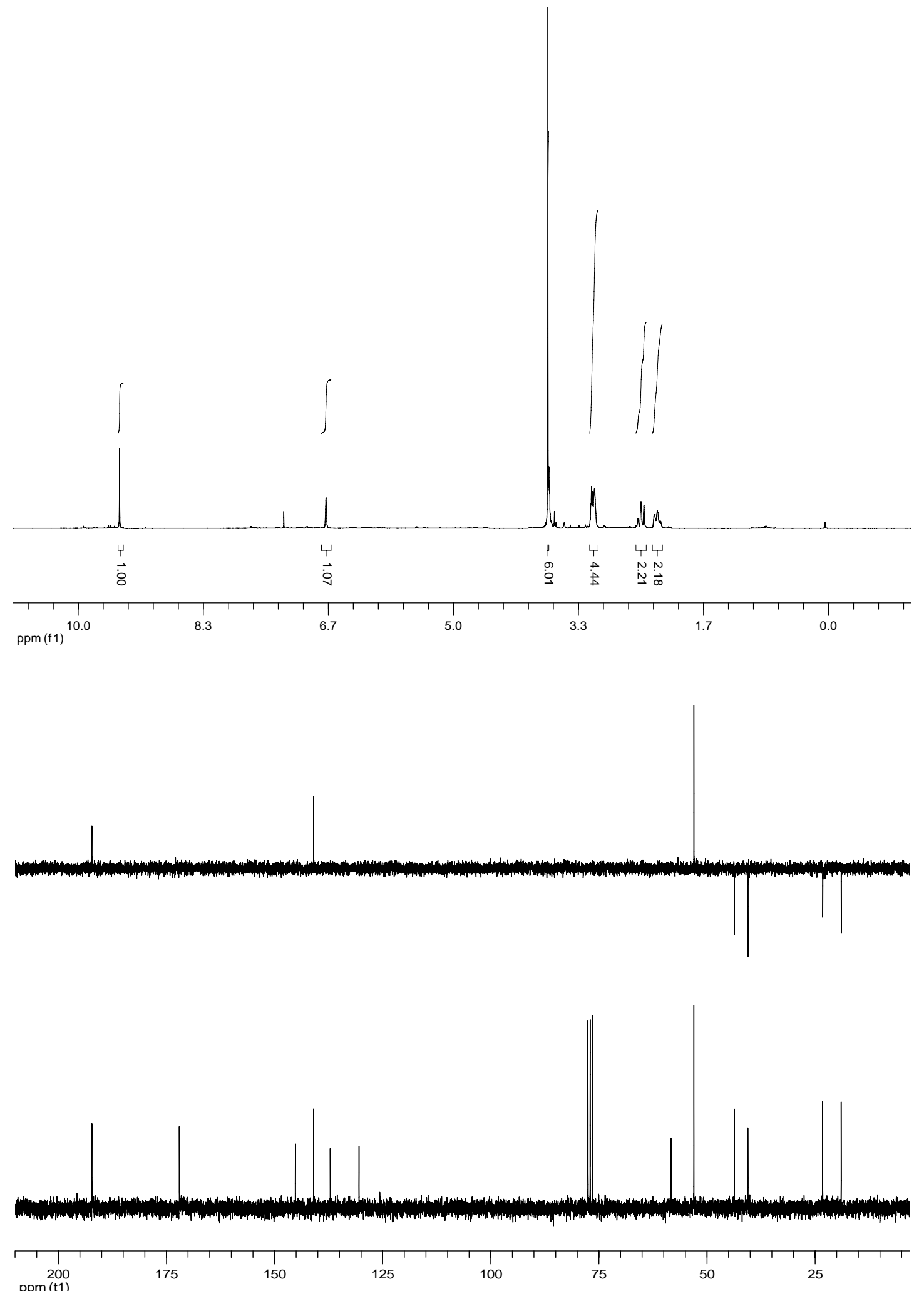
ppm (t1) 
S20
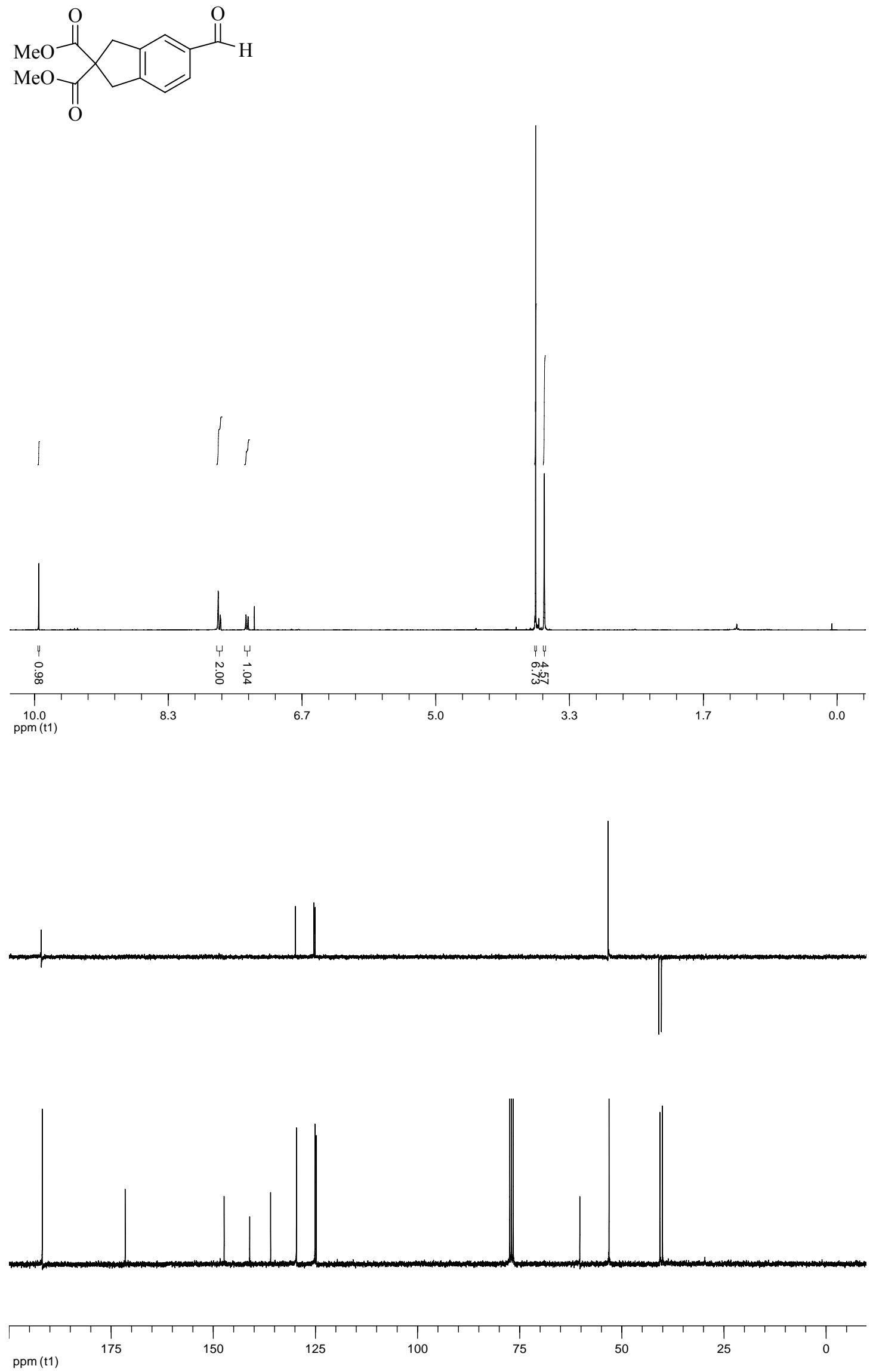

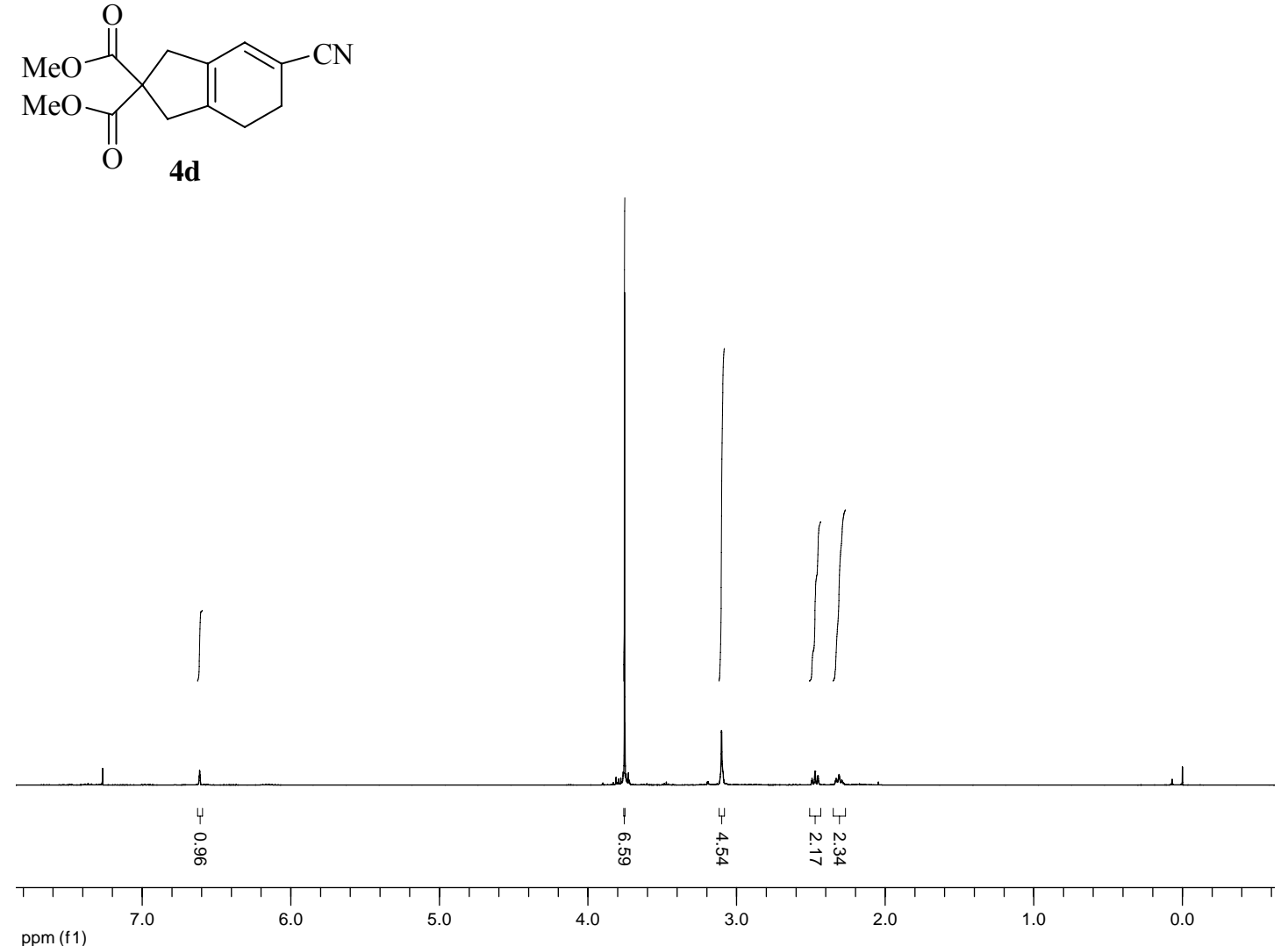

ppm (f1)
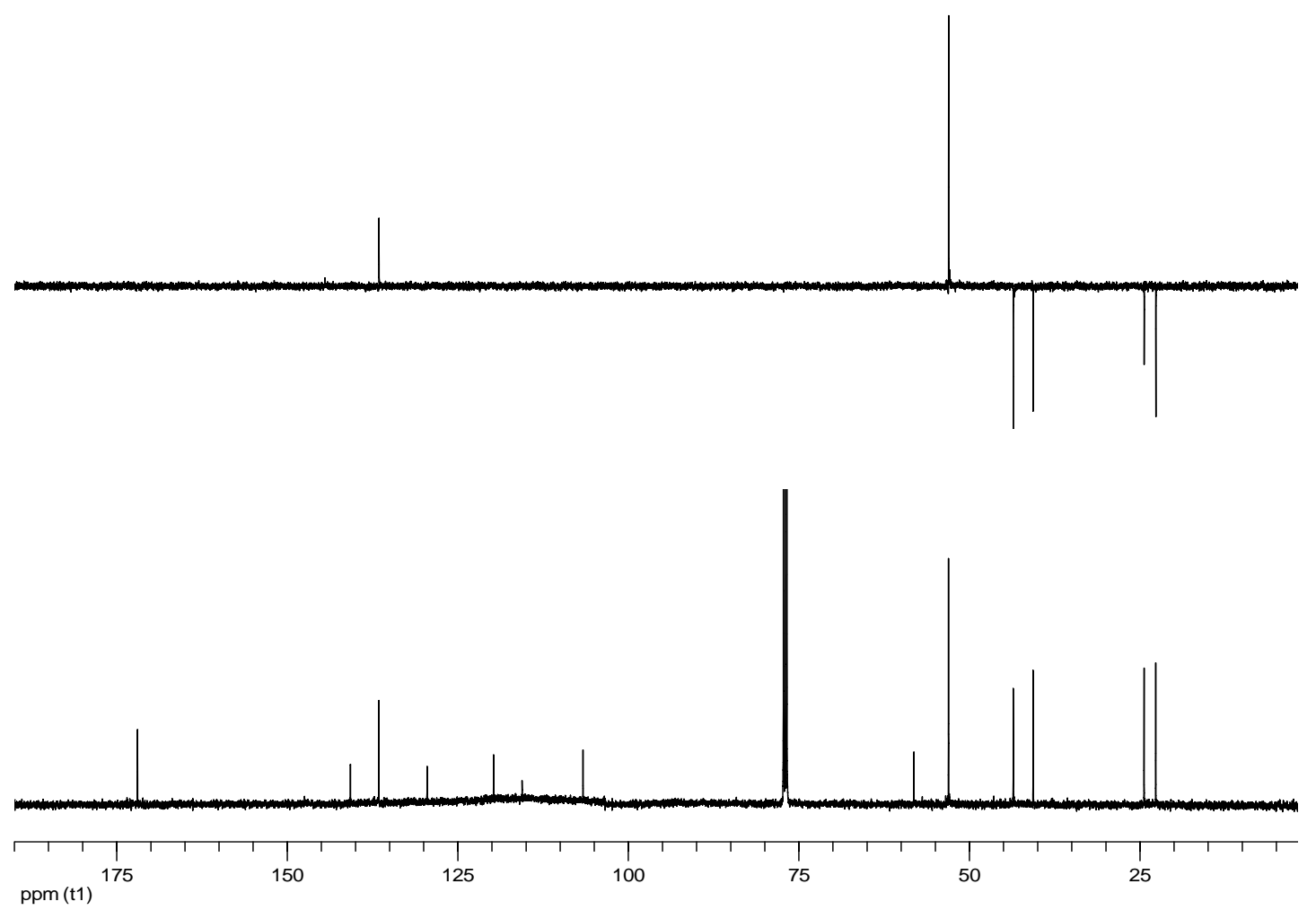


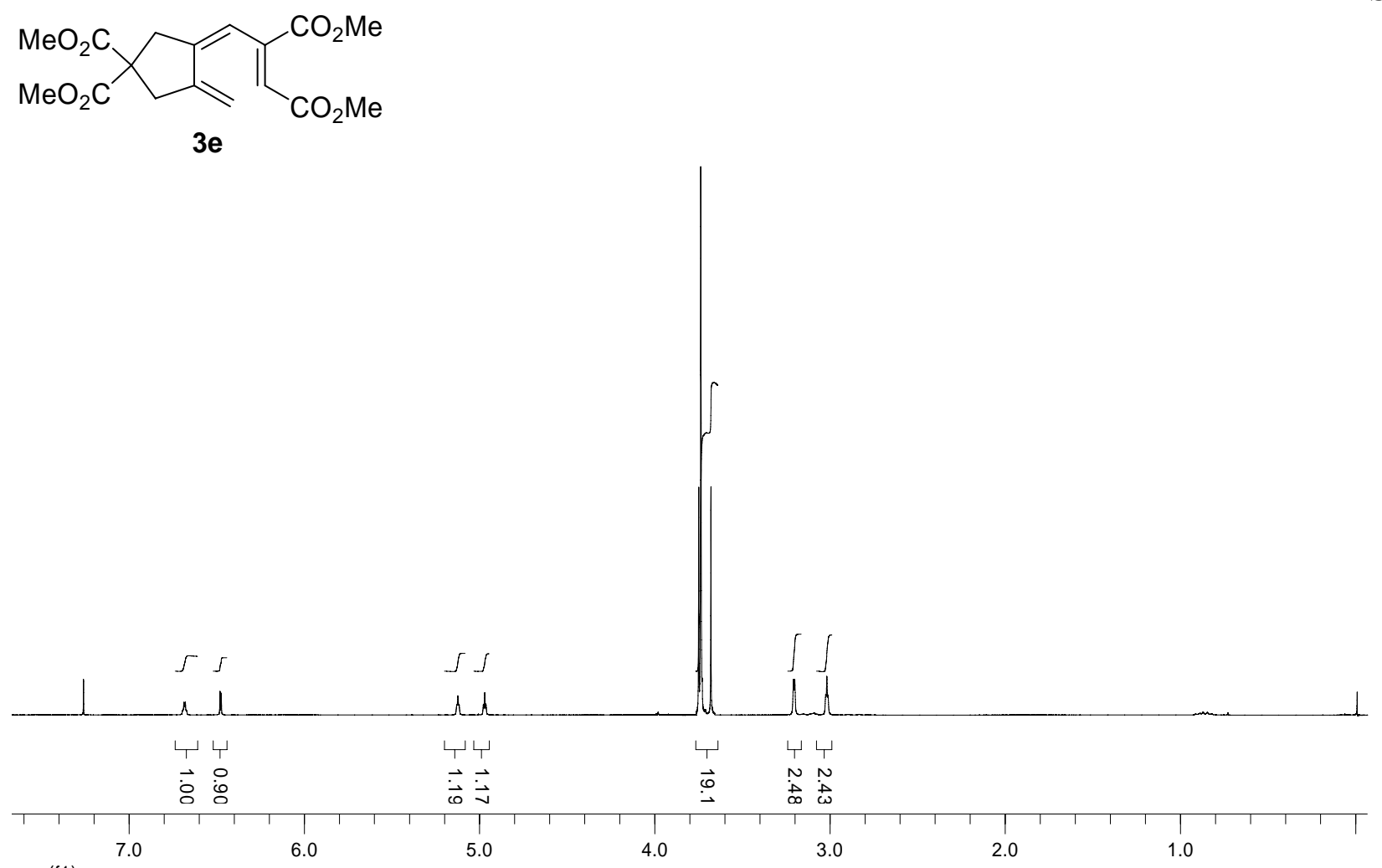

Jpm (f1)

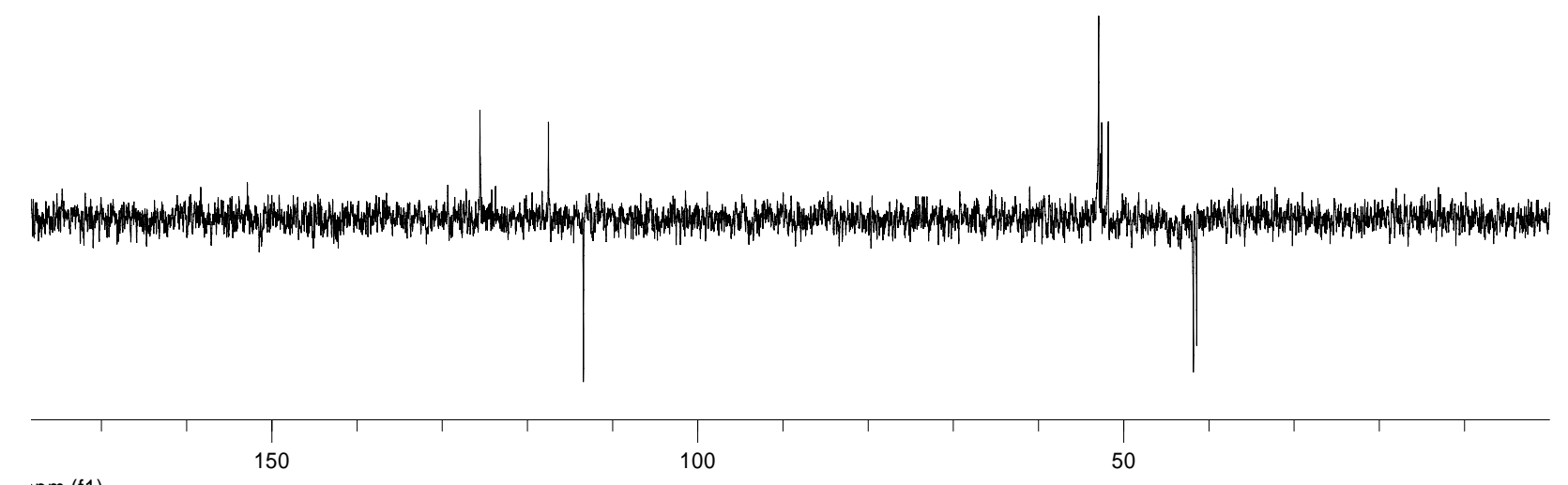

ıpm (f1)

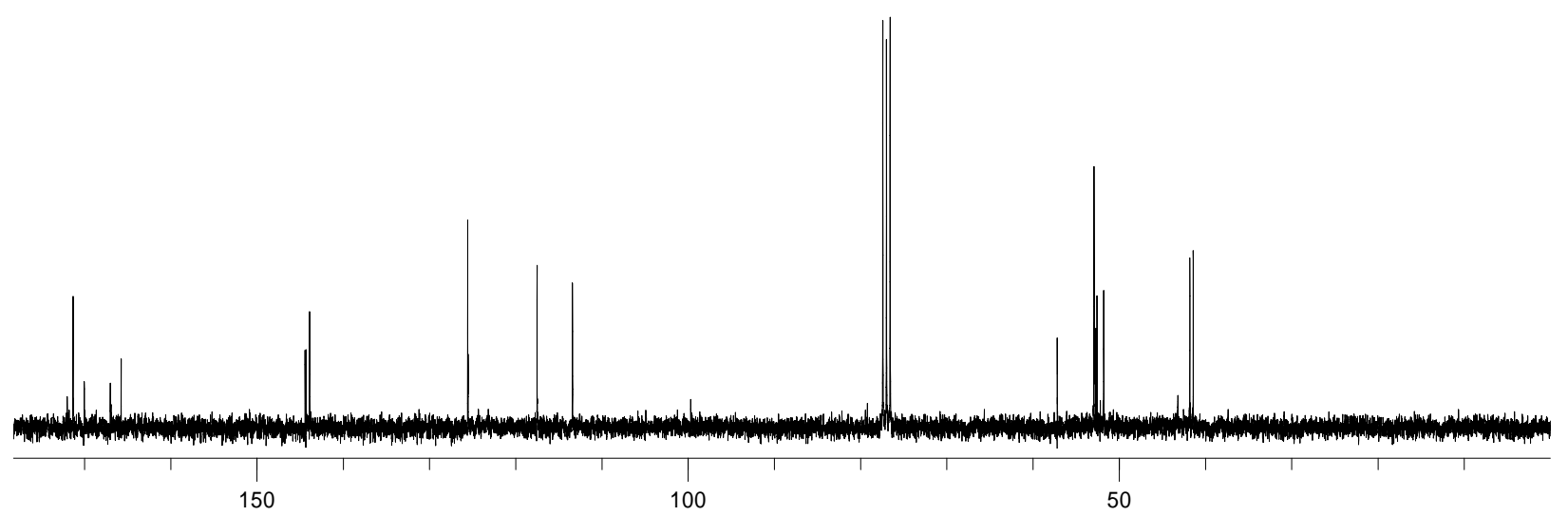

ıpm (f1) 

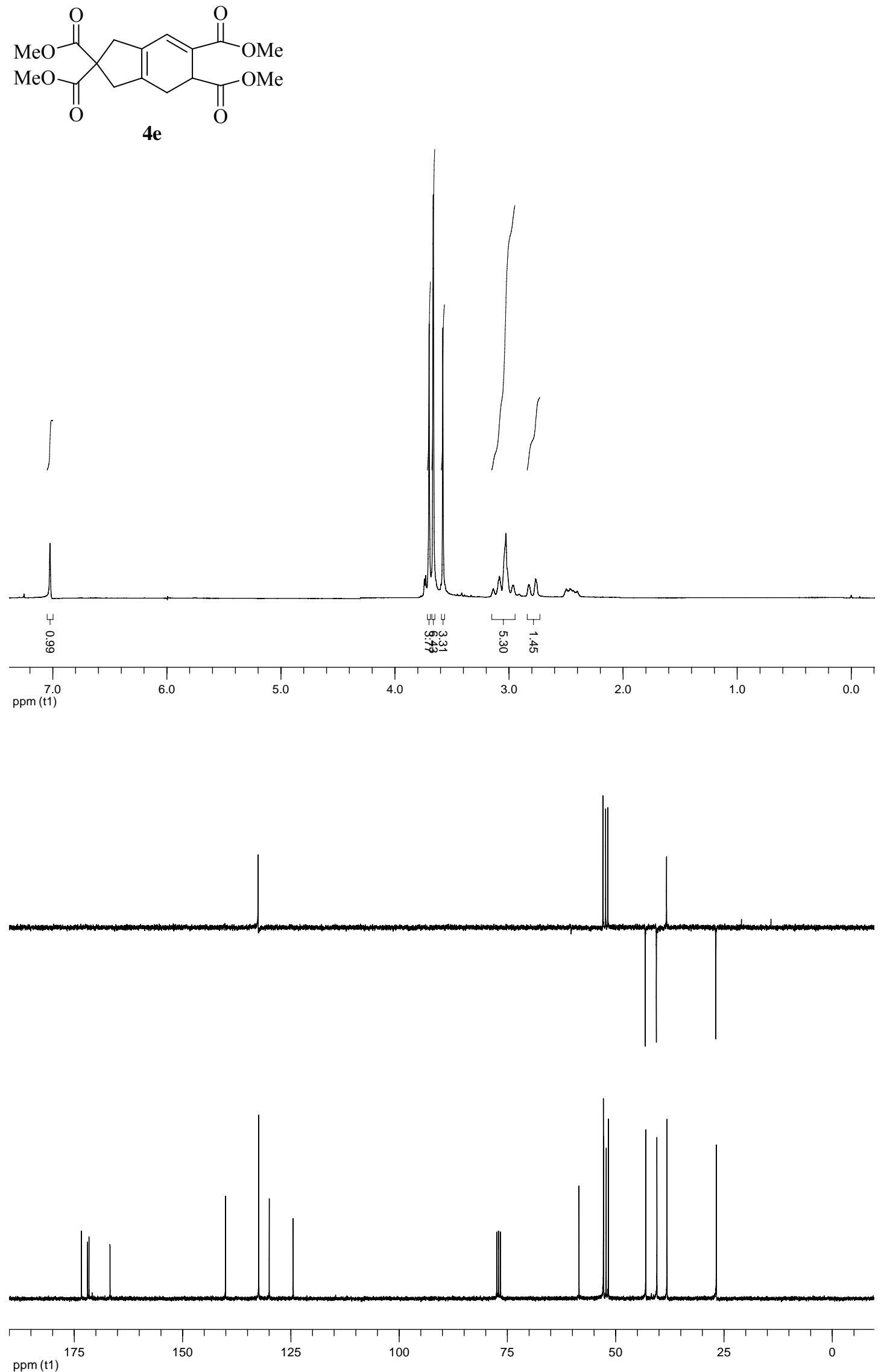

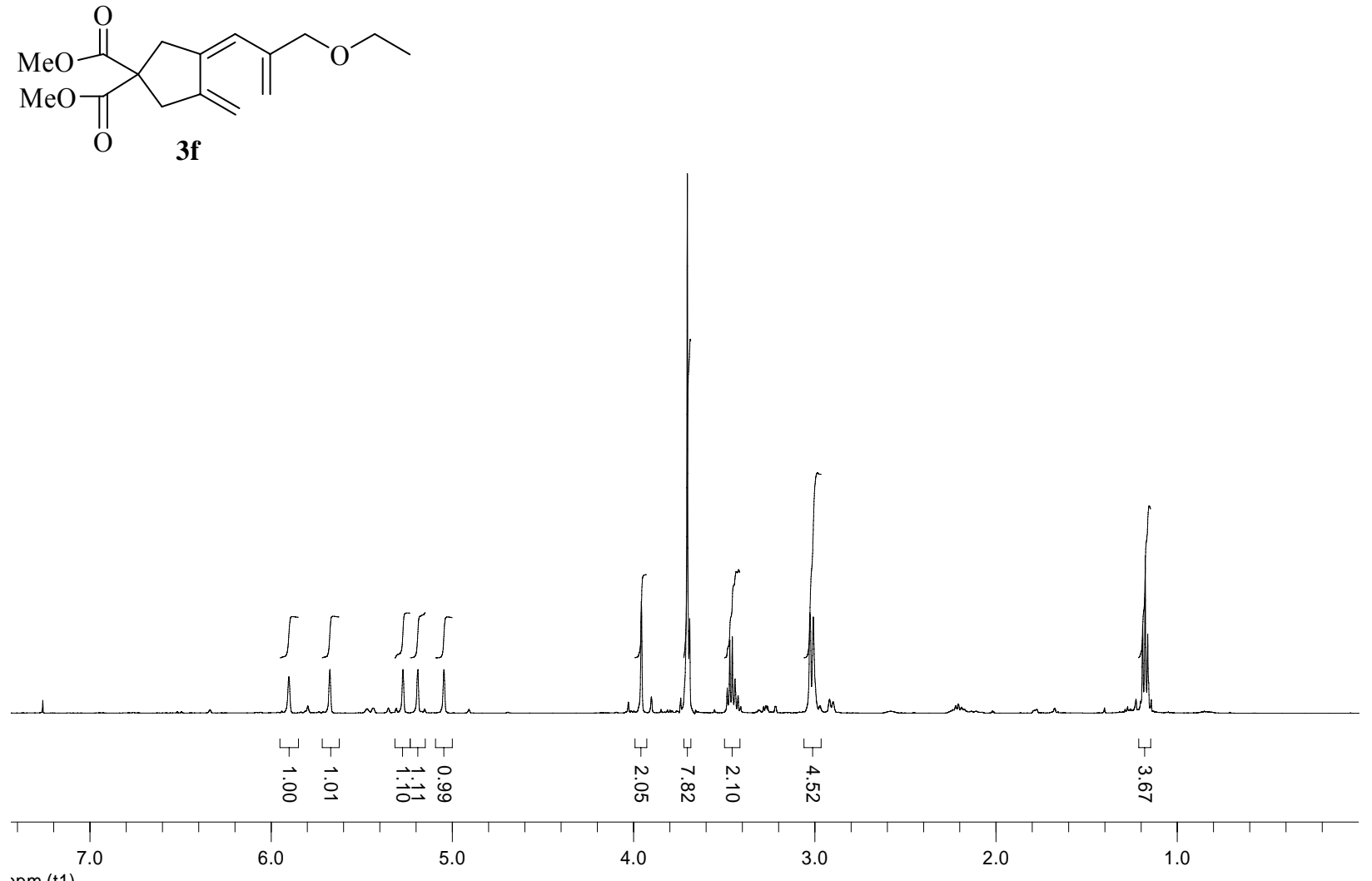

.jpm (t1)
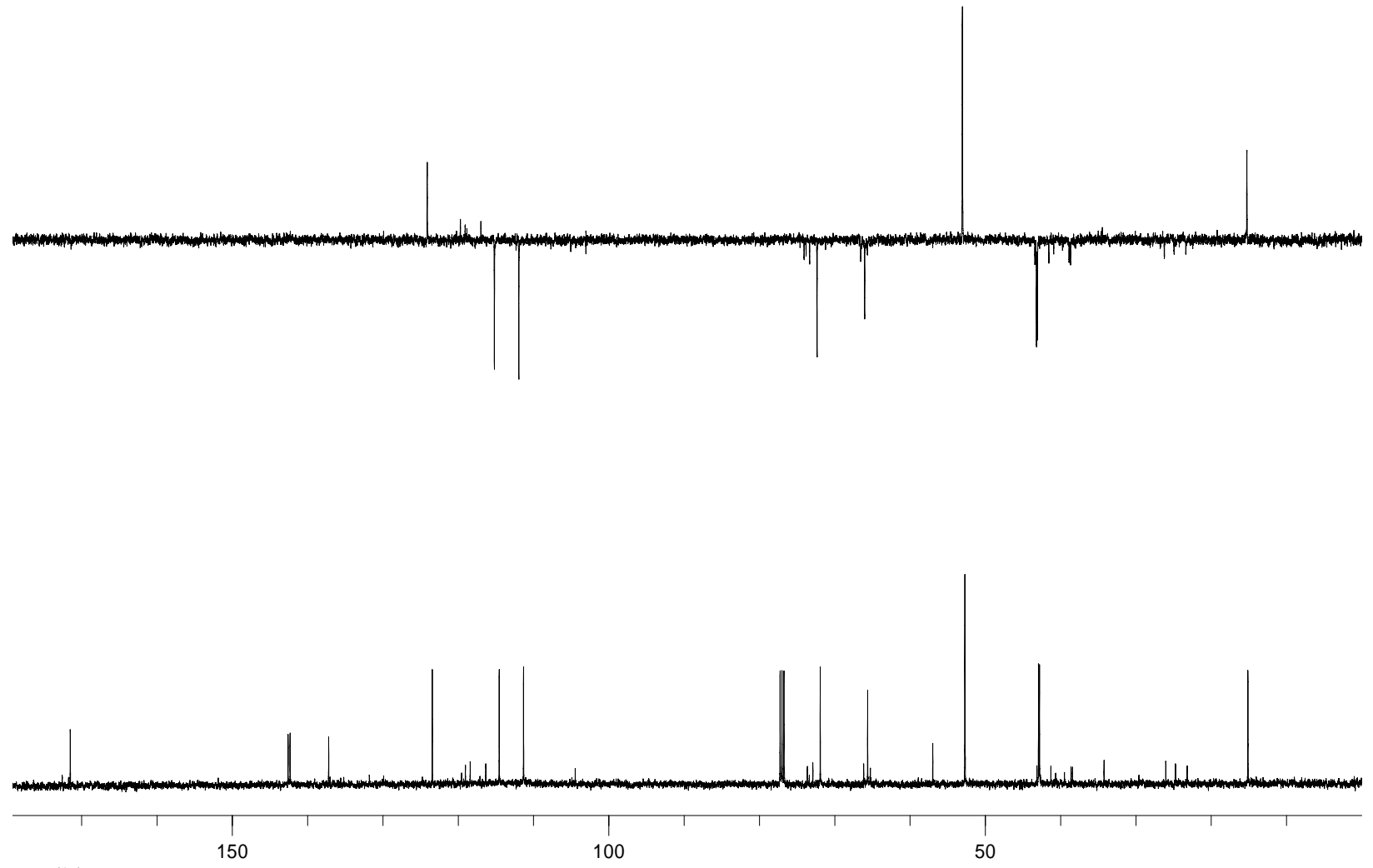

ıpm (f1) 

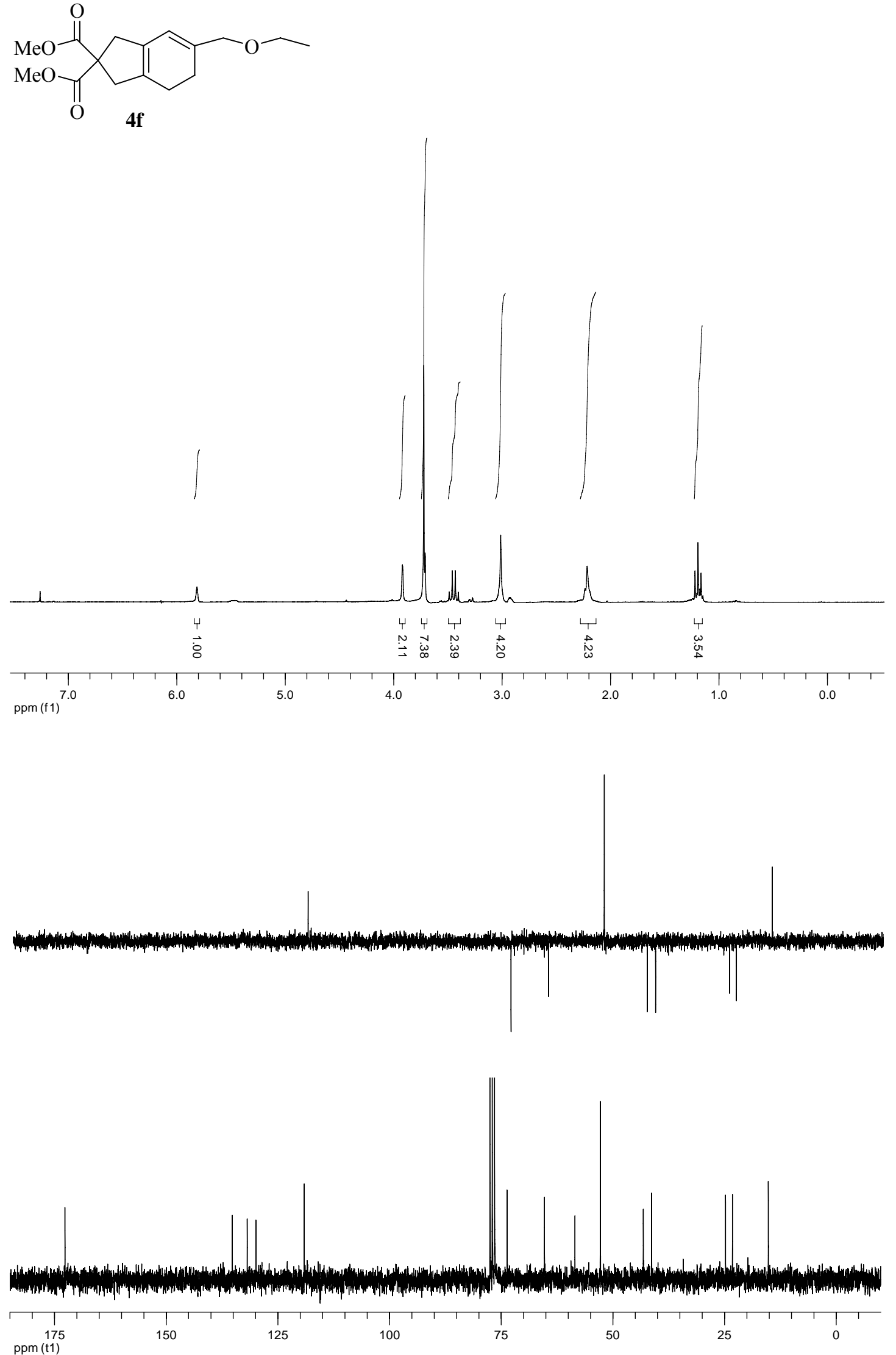
<smiles>COC(=O)C1(C(=O)OC)CC2=C(CCC(COc3ccccc3)=C2)C1</smiles>

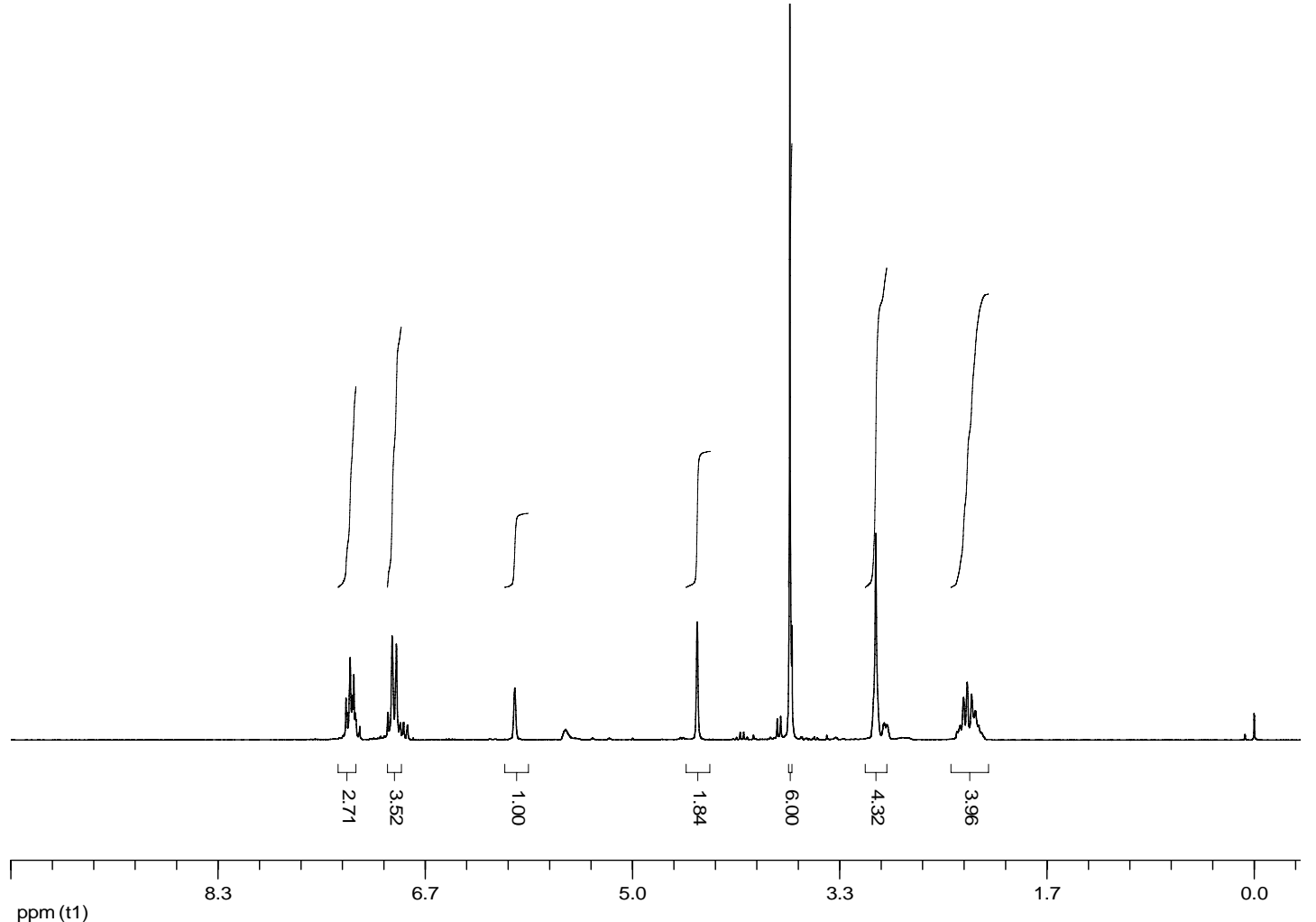

ppm (t1)

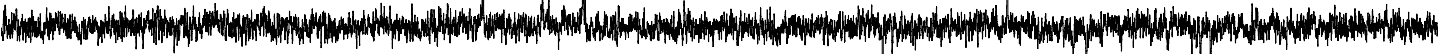

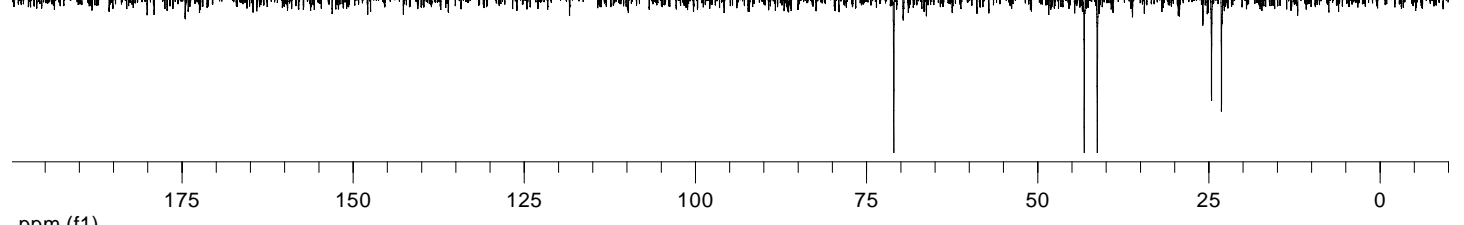

ppm (f1)

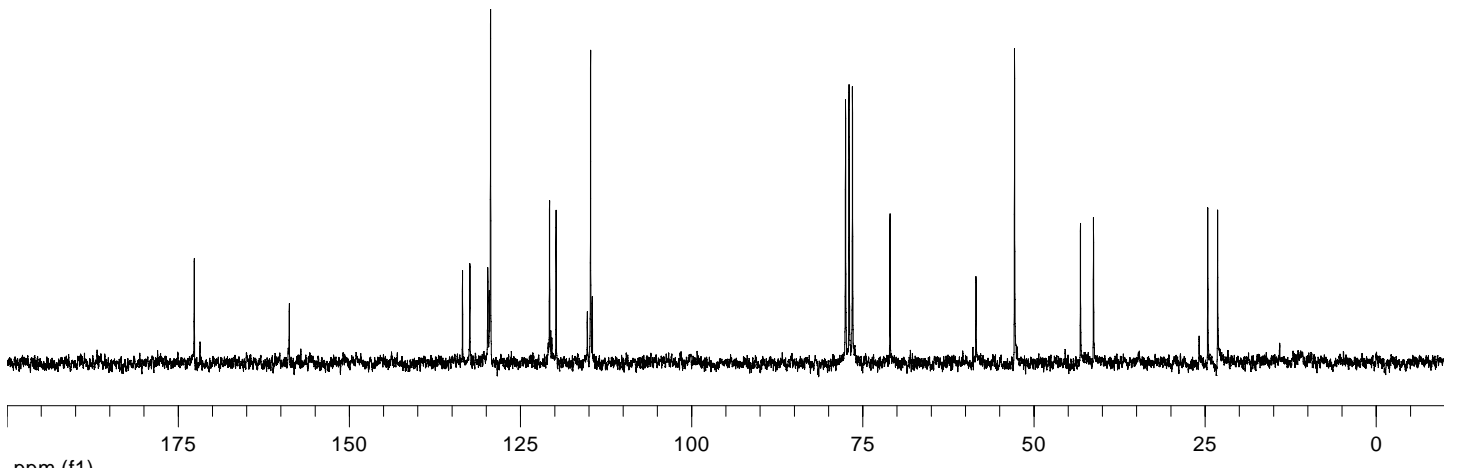

ppm (f1) 
<smiles>COCC1=CC2=C(CC1)CC(C(=O)OC)(C(=O)OC)C2</smiles>

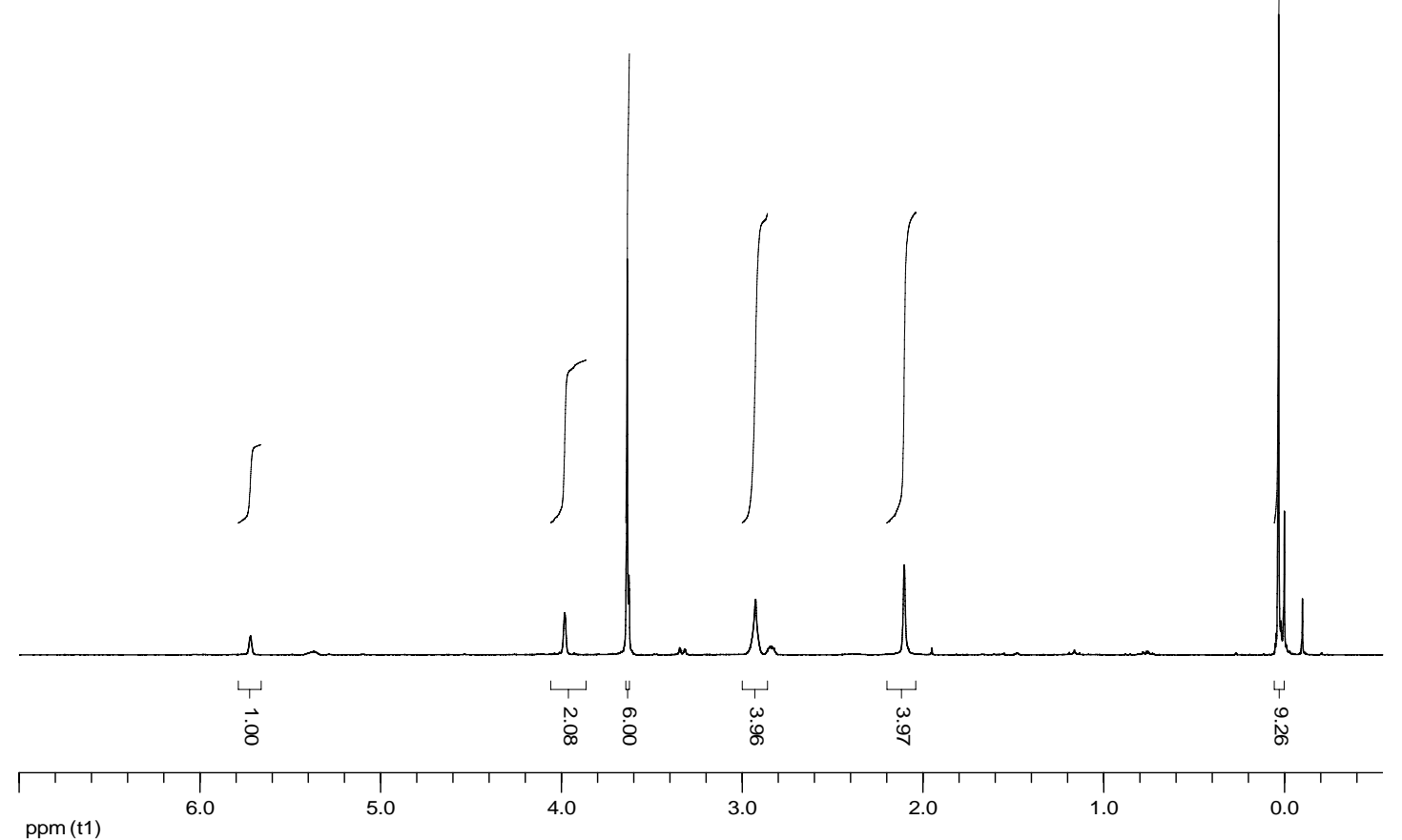

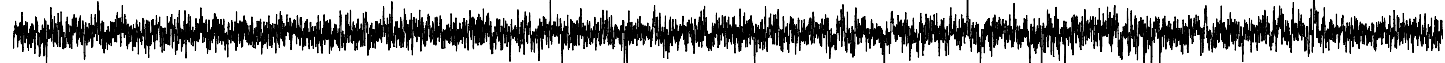

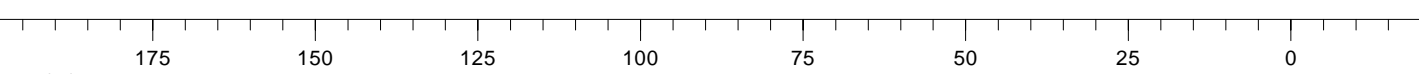
ppm (t1)

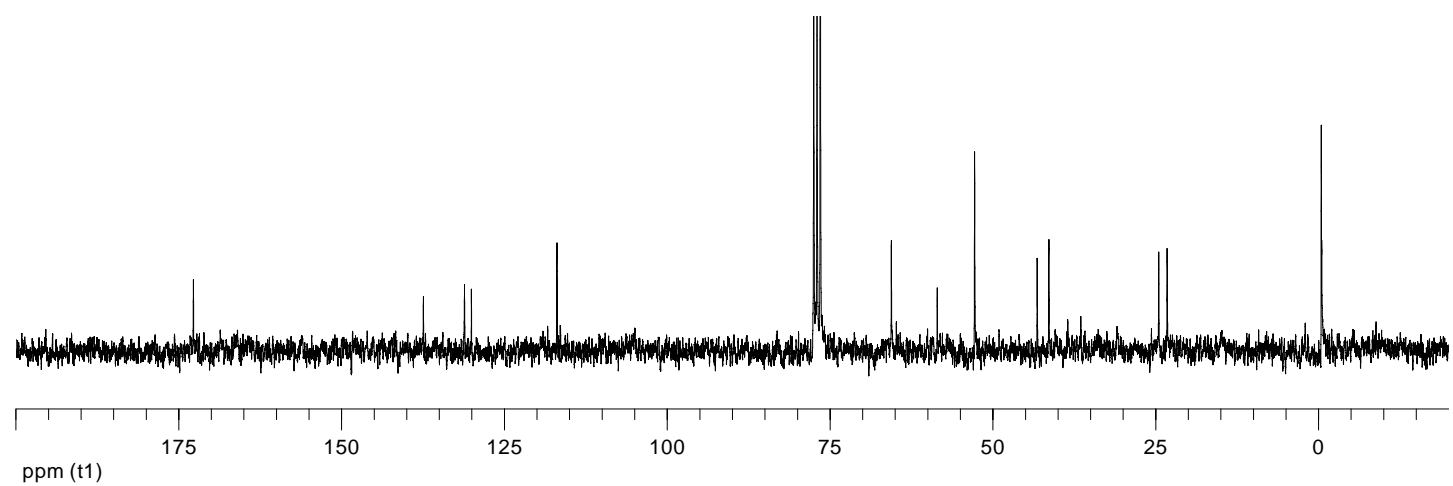


S28
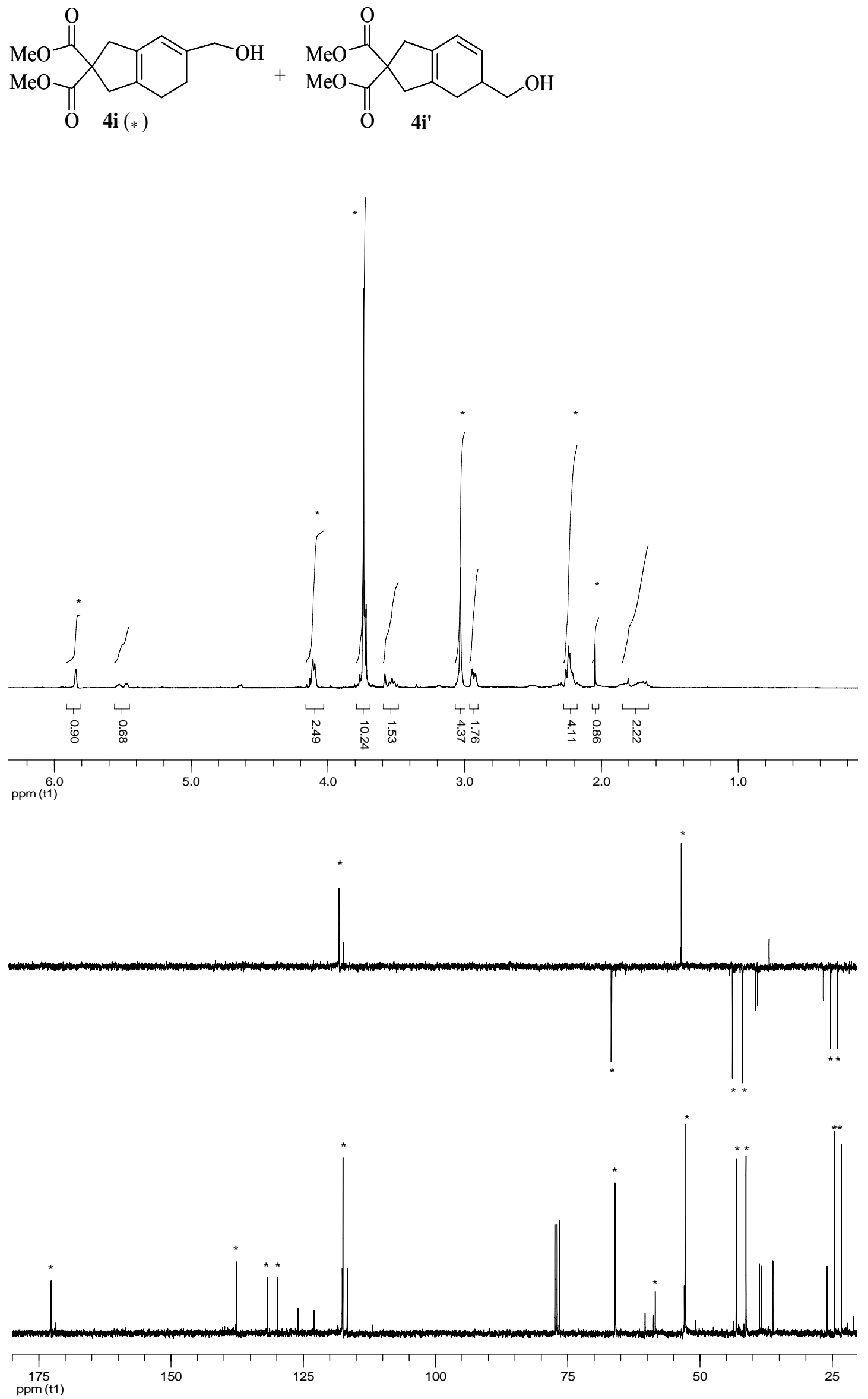

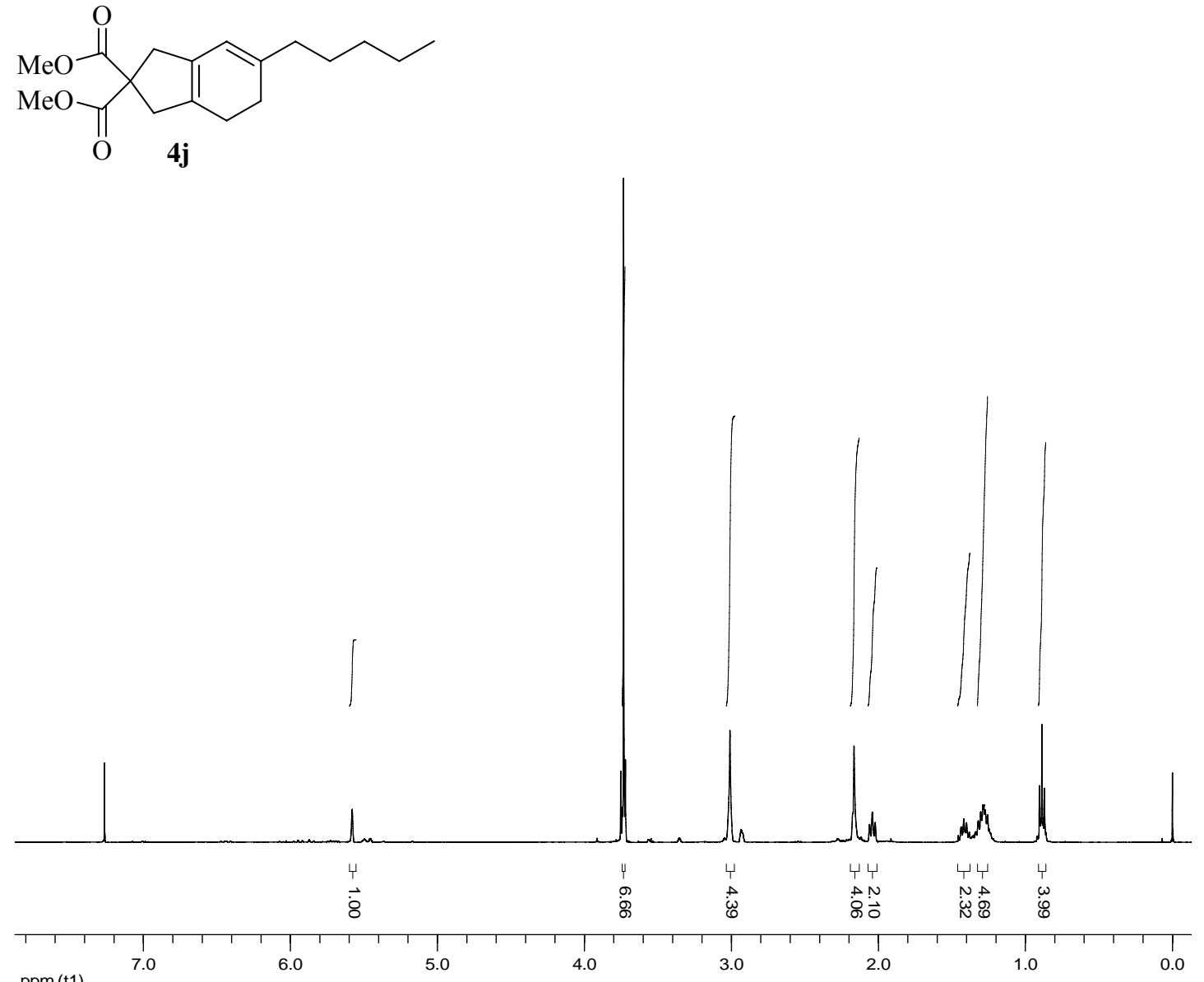

ppm (t1)
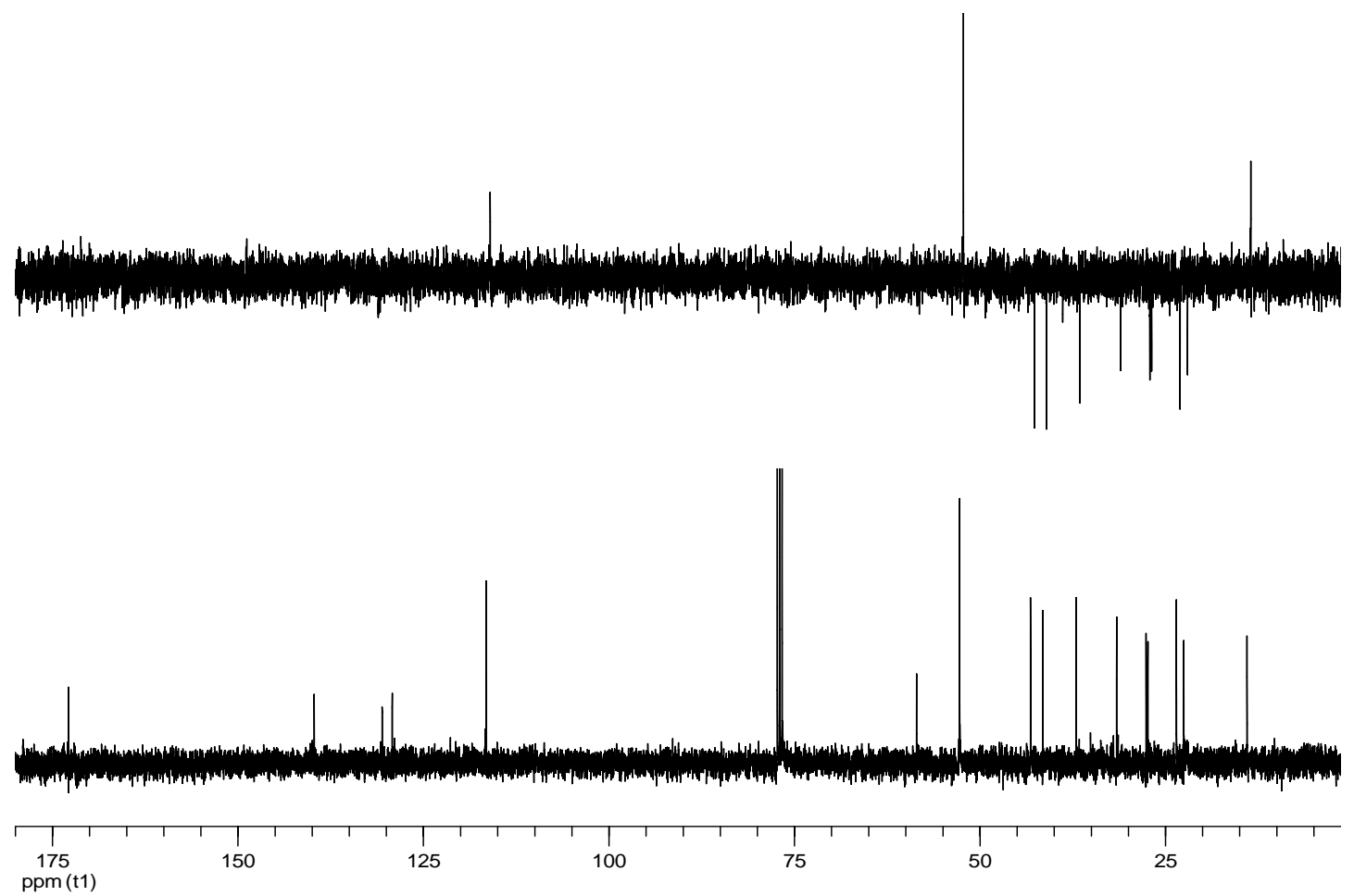
<smiles>COC(=O)C1(C(=O)OC)CC2=C(CCC(C(C)(C)C)=C2)C1</smiles>
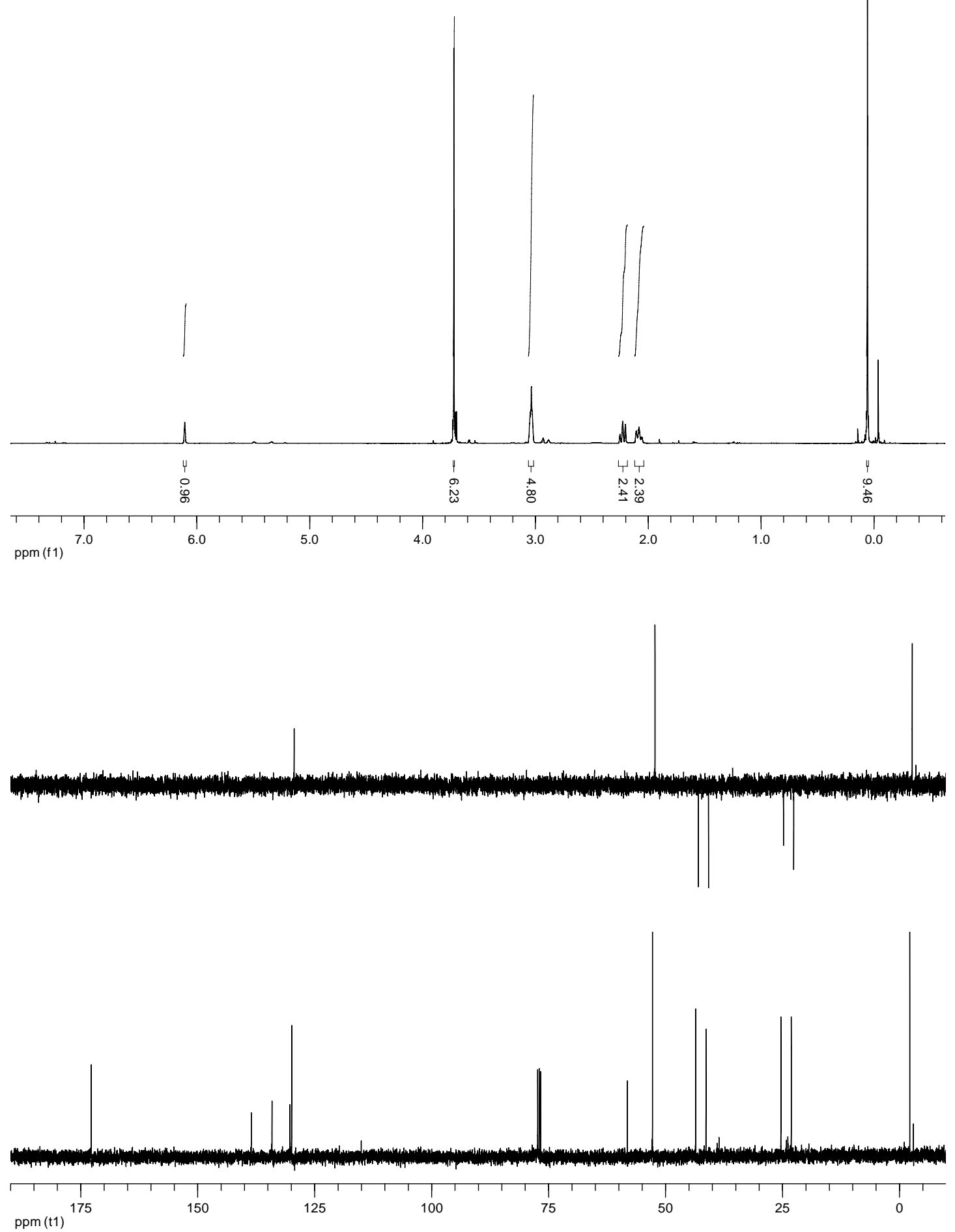

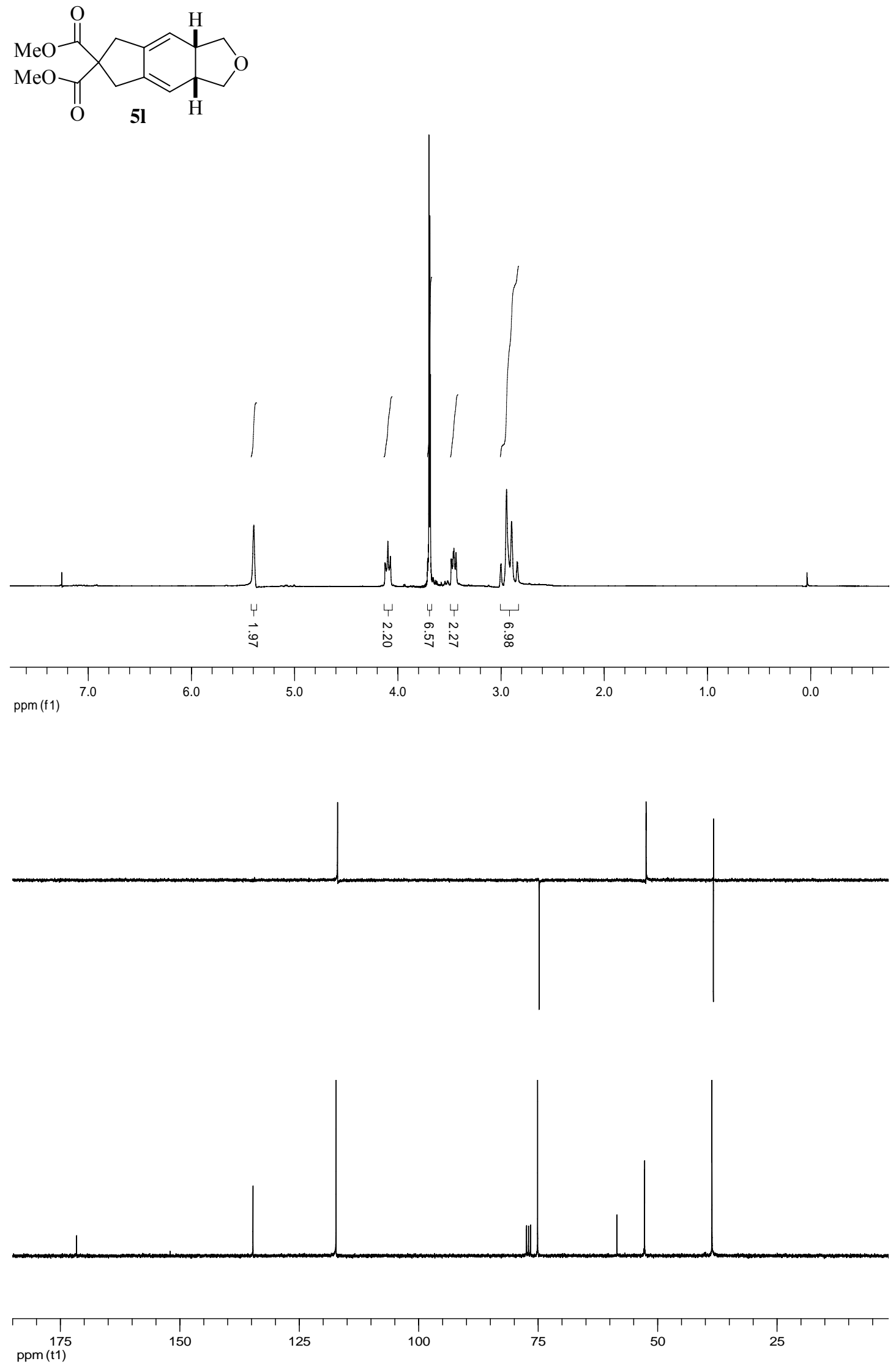
S32

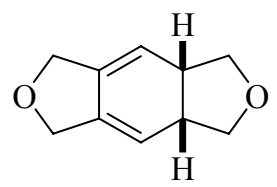

5l'
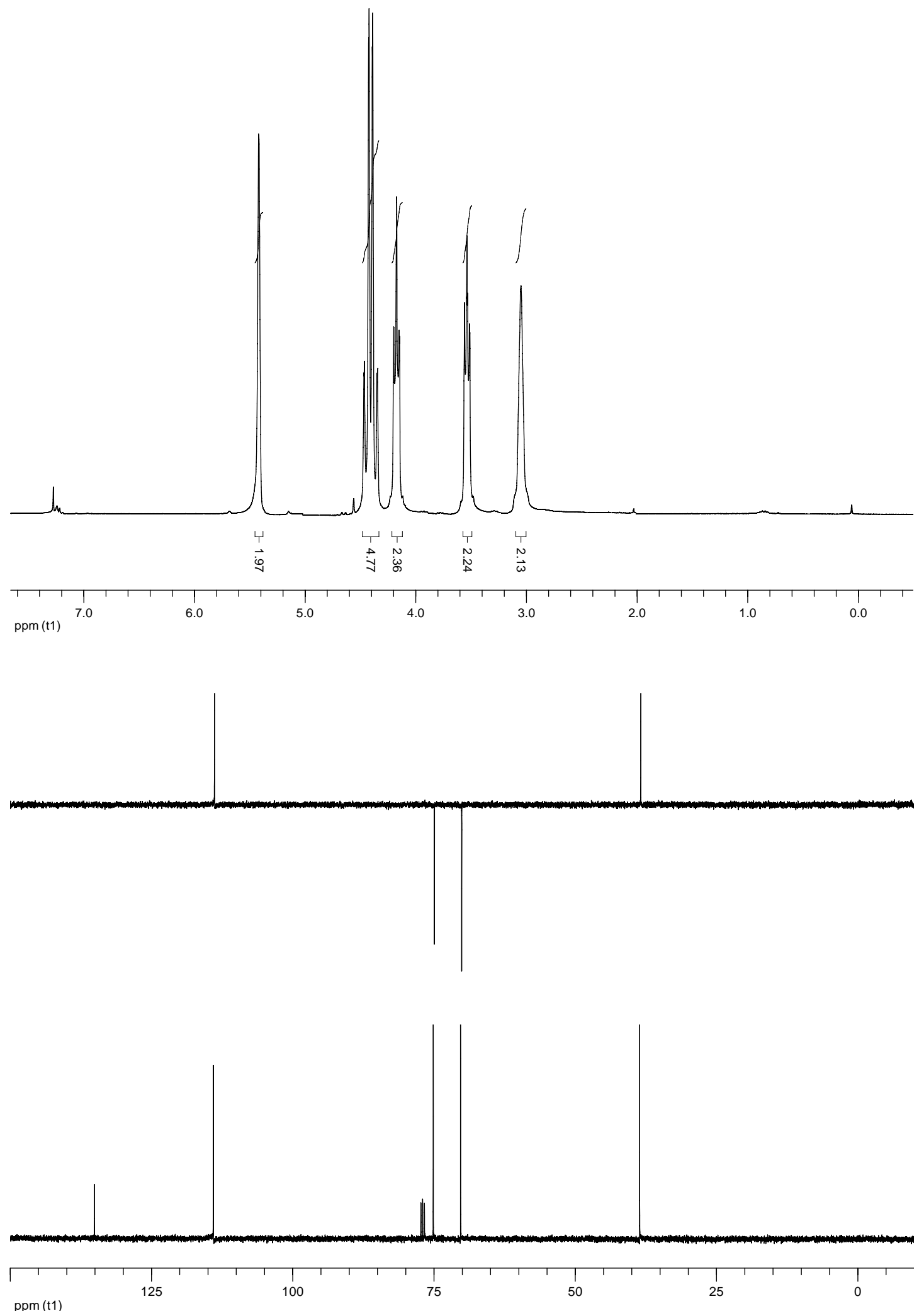

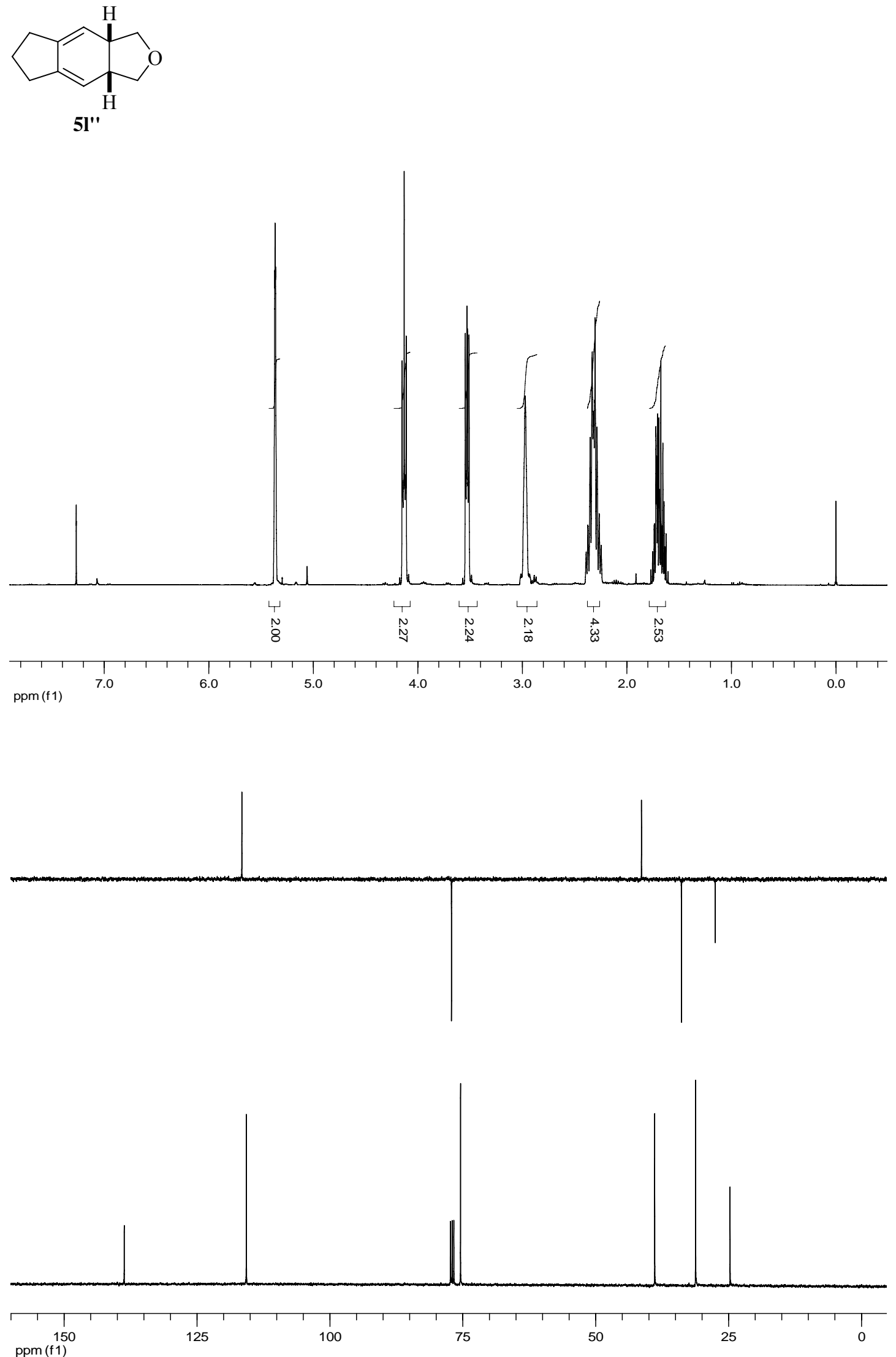

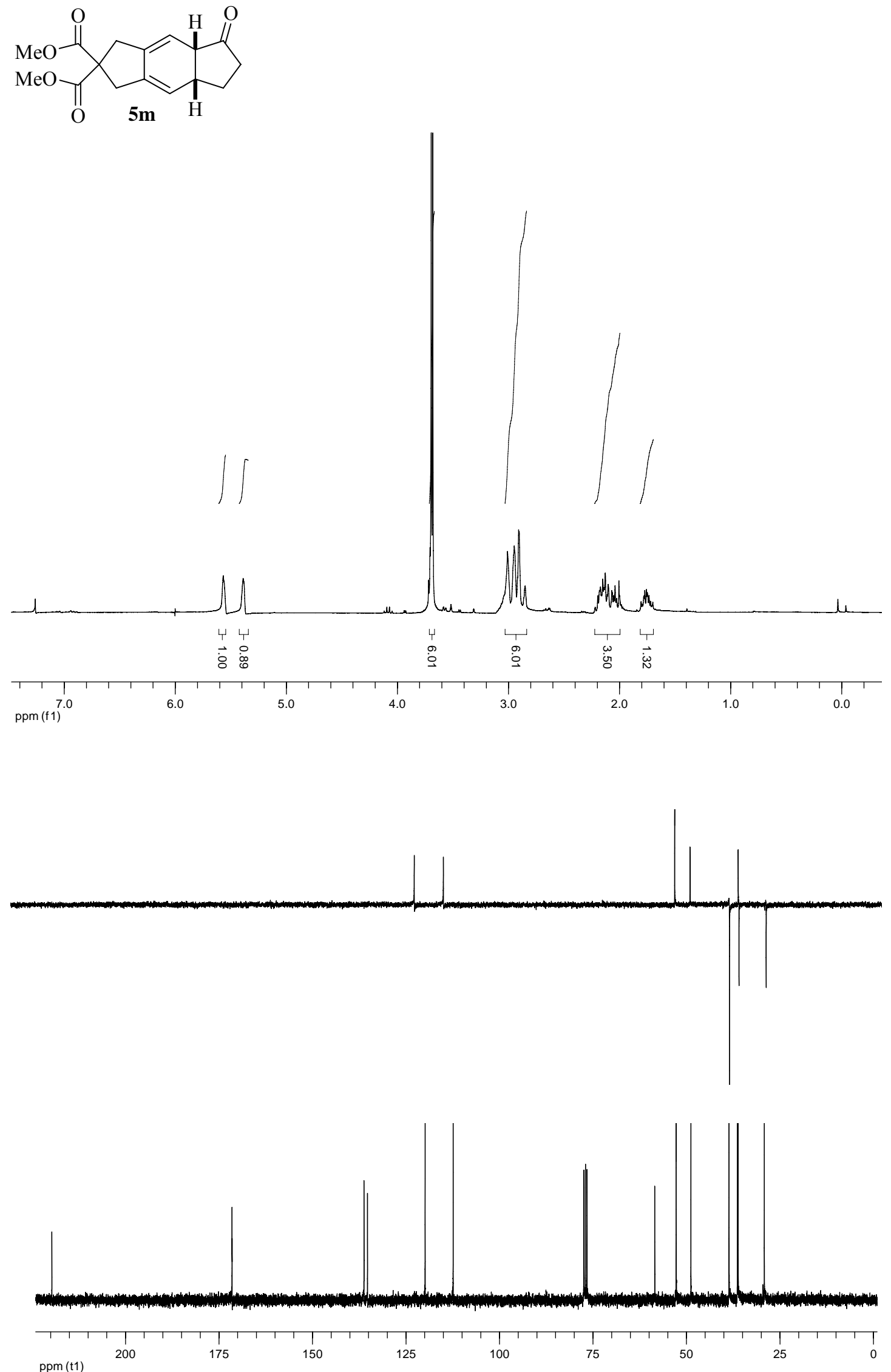
S35
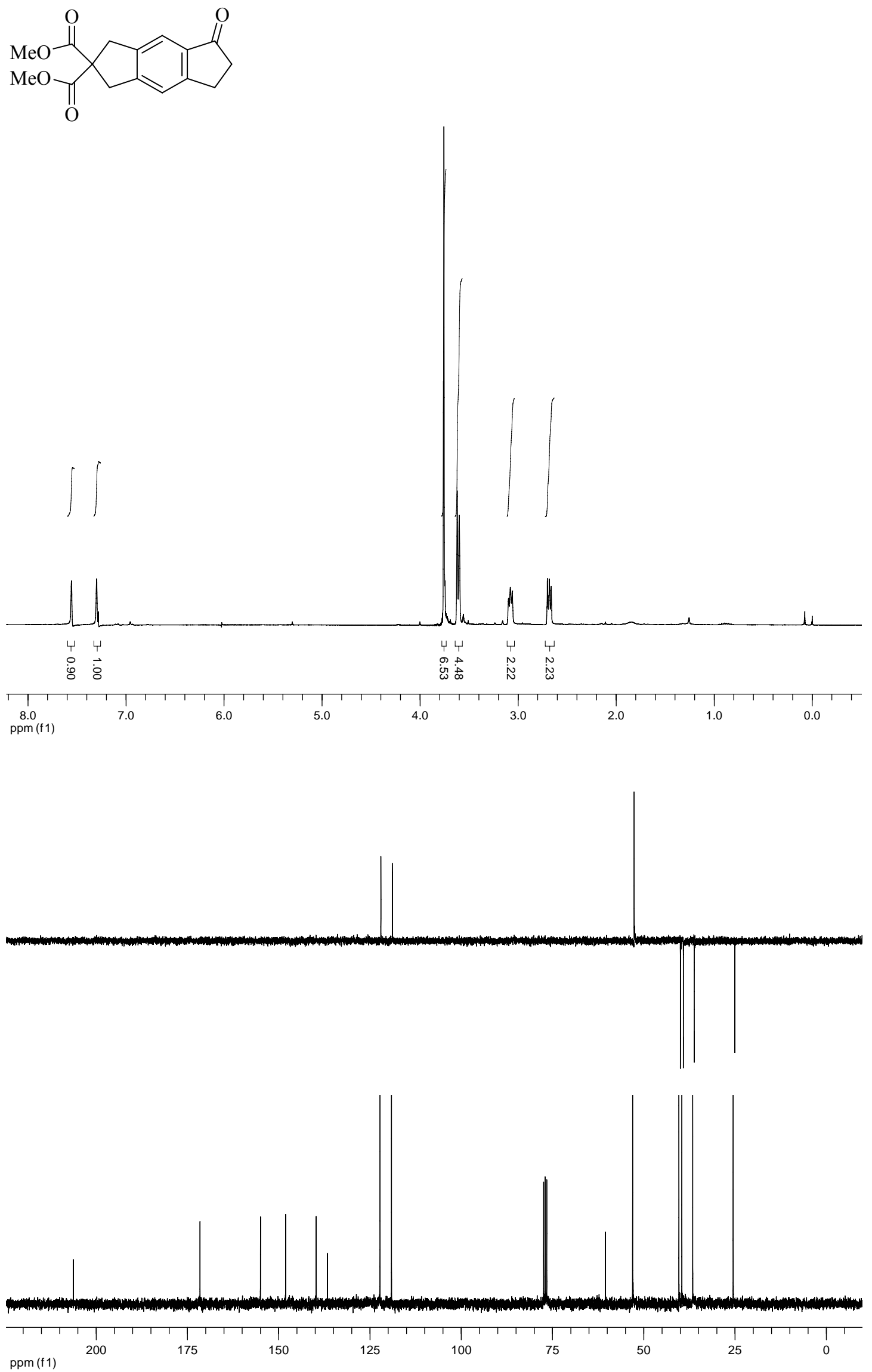
<smiles>COC(=O)C1(C(=O)OC)Cc2cc3c(cc2C1)C(=O)C=CC3=O</smiles>

$5 n$

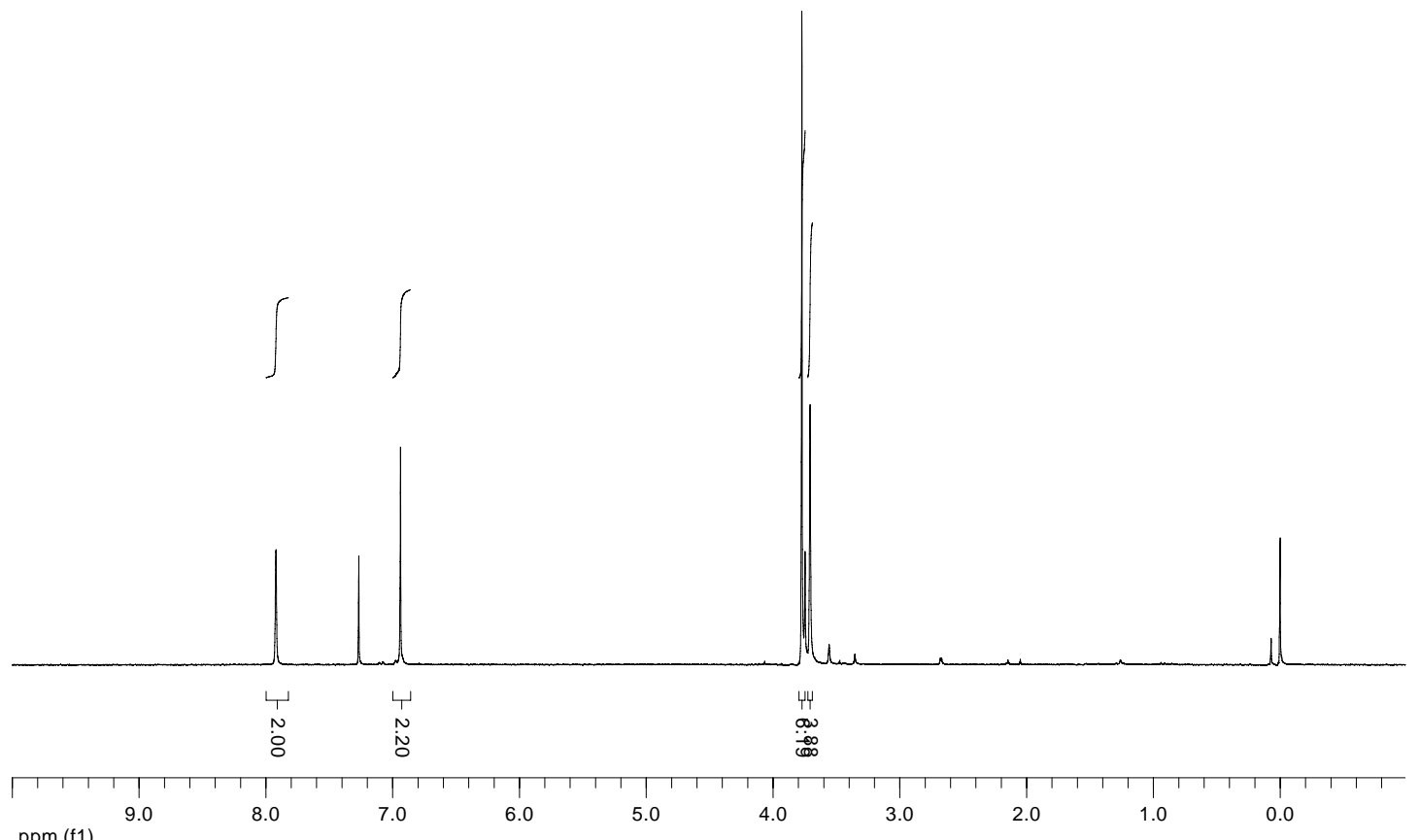

ppm (f1)

(1)

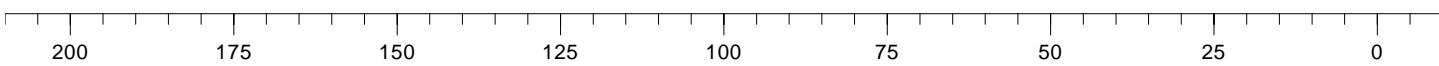

ppm (f1)

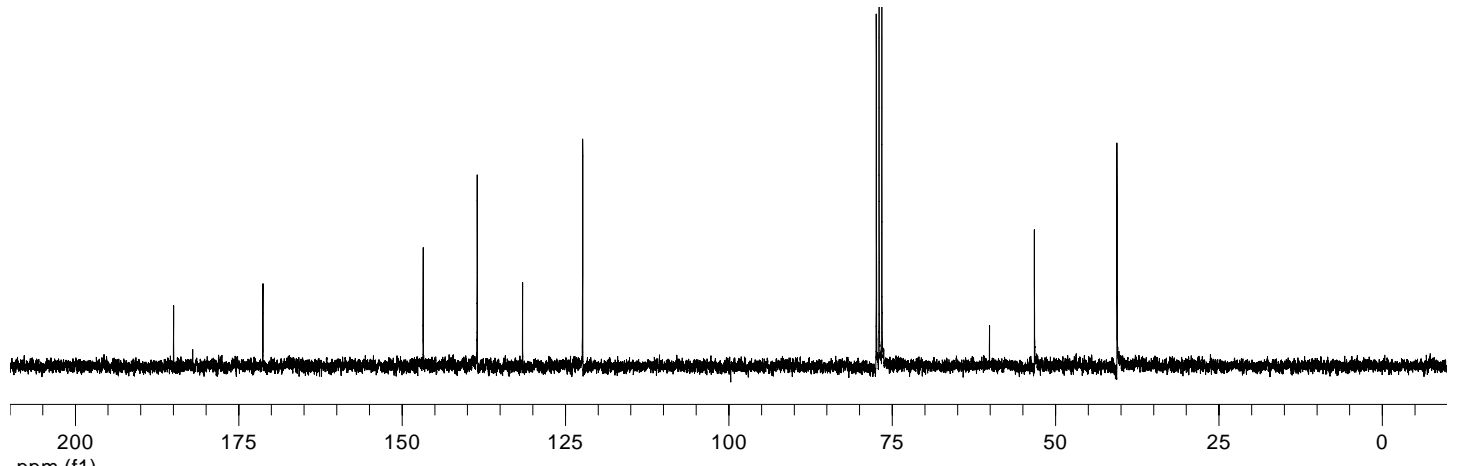

ppm (f1) 

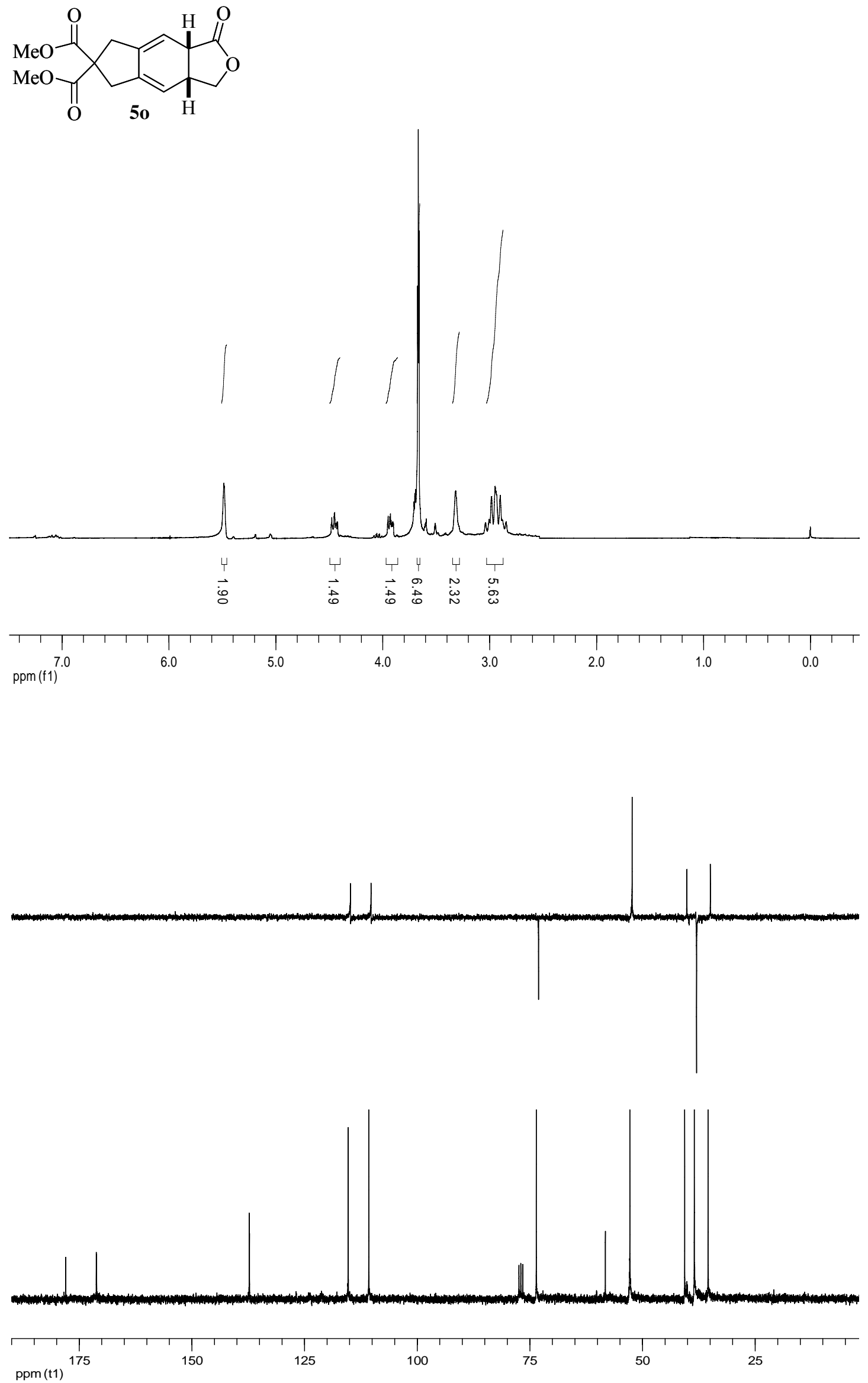
<smiles>COC(=O)C1(C(=O)OC)CC2=C[C@H]3CCC[C@H]3C=C2C1</smiles>
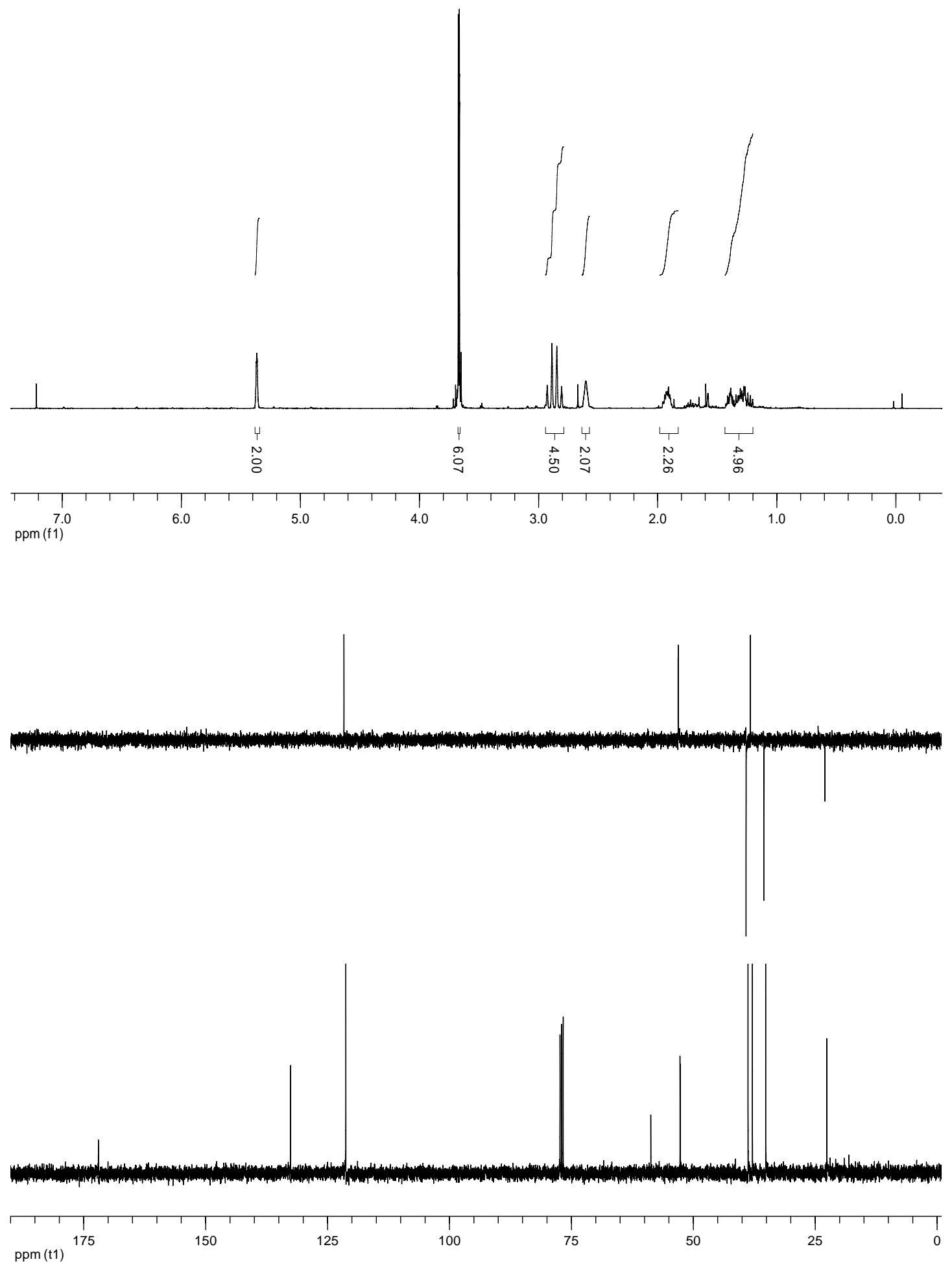

ppm (t1) 

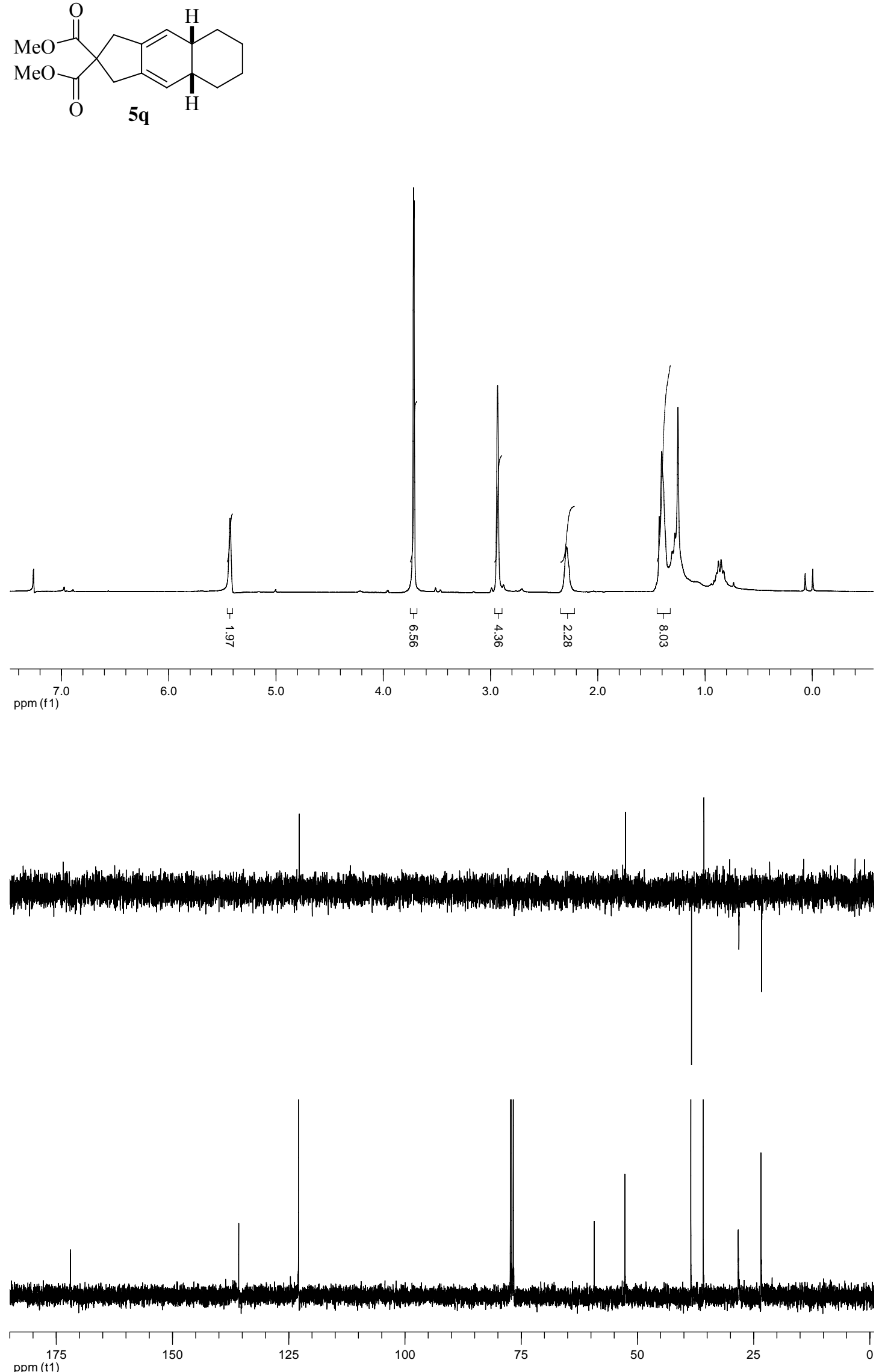